\title{
CONSUMER SEARCH COSTS AND THE INCENTIVES TO MERGE UNDER BERTRAND COMPETITION
}

\author{
José L. Moraga-González
}

Vaiva Petrikaite 
The Public-Private Sector Research Center is a Research Center based at IESE Business School. Its mission is to develop research that analyses the relationships between the private and public sectors primarily in the following areas: regulation and competition, innovation, regional economy and industrial politics and health economics.

Research results are disseminated through publications, conferences and colloquia. These activities are aimed to foster cooperation between the private sector and public administrations, as well as the exchange of ideas and initiatives.

The sponsors of the SP-SP Center are the following:

- Accenture

- Ajuntament de Barcelona

- Departament d' Economia i Coneixement de la Generalitat de Catalunya

- Departament d' Empresa i Ocupació de la Generalitat de Catalunya

- Diputació de Barcelona

- Endesa

- Fundació AGBAR

- Institut Català de les Indústries Culturals

- Mediapro

- Sanofi Aventis

- ATM, FGC y TMB

The contents of this publication reflect the conclusions and findings of the individual authors, and not the opinions of the Center's sponsors. 


\title{
Consumer Search Costs and the Incentives to Merge under Bertrand Competition
}

\author{
José L. Moraga-González1 Vaiva Petrikaitë*
}

First version: March 2009

Revised: July 2011

\begin{abstract}
This paper studies the incentives to merge in a Bertrand competition model where firms sell differentiated products and consumers search the market for satisfactory deals. In the pre-merger market equilibrium, all firms look alike and so the probability a firm is next in the queue consumers follow when visiting firms is equal across non-visited firms. However, after a merger, insiders raise their prices more than the outsiders so consumers search for good deals first at the non-merging stores and then, if they do not find any product satisfactory enough, they continue searching at the merging stores. When search cost are negligible, the results of Deneckere and Davidson (1985) hold. However, as search costs increase, the merging firms receive fewer customers so mergers become unprofitable for sufficiently large search costs. This new merger paradox is more likely the higher the number of non-merging firms.
\end{abstract}

Keywords: mergers, search, insiders, outsiders, order of search

JEL Classification: D40, D83, L13

${ }^{1}$ Professor of Economics, IESE

${ }^{*}$ We thank Heski Bar-Isaac, Allard van der Made, Marielle Non, Zsolt Sándor and specially Jidong Zhou for their useful comments. This paper has also benefited from presentations at IESE Business School, University Pompeu Fabra, the IIOC 2009 in Boston, the EARIE 2009 meetings in Liubljana and the 1st Groningen Workshop on Search and Switching Costs (December 2009). Financial support from the Marie Curie Excellence Grant MEXT-CT-2006-042471 is gratefully acknowledged. Moraga (jose.l.moraga@gmail.com): ICREA, IESE and U. Groningen. Petrikaitè (v.petrikaite@rug.nl): University of Groningen. 


\section{Introduction}

One of the most important insights in merger analysis is that merging is not very attractive in environments where firms compete in quantities and offer similar products (Salant et al., 1983). This result, known as the merger paradox, arises because the output reduction of the merging firms, which favors the coalition partners, is accompanied by an output expansion of the non-merging ones, which hurts them and has a dominating influence. Deneckere and Davidson (1985) show that price-setting firms selling horizontally differentiated products, other things equal, always have an incentive to merge. This result arises because price increases of the merging firms, which favor the coalition partners, are accompanied by price increases of the non-merging firms, which also favors them.

While no one would deny that searching for price and product fit is costly in real-world markets -think for example about the time we spend test-driving new cars, acquiring new furniture, trying on new clothes, etc.- there has been little work in the industrial organization literature on the influence of search costs on the incentives to merge and on the aggregate implications of mergers. In this paper we argue that when search costs are important mergers may become unprofitable, even if they involve firms competing in prices and selling horizontally differentiated products.

Our model has a finite number of firms selling differentiated products. The exact utility a buyer

derives from consuming a product can only be ascertained upon visiting the seller. Consumers search for satisfactory deals sequentially. Firms compete in prices. In the pre-merger market, all firms look alike and when consumers pick a first shop to visit, they do so in a random way. Those consumers who fail to find a satisfactory product continue searching and once again they pick the next shop to visit randomly; and so on. This model was introduced by Wolinsky (1986) and was further studied by Anderson and Renault (1999). When search cost is equal to zero, the model is similar to Perloff and Salop (1985) and merger analysis gives the same results as in Deneckere and Davidson (1985).

When search cost is positive, higher prices charged by the merging stores result in consumers searching first at the non-merging firms' and then, in the event they fail to find a satisfactory product in those firms, continue searching at the merging stores. In equilibrium, as search costs increase, the share of consumers who walk away from the non-merging stores and show up at the merged shops falls, which makes merging less profitable. We show that any 2-firm merger is unprofitable if search costs are sufficiently high. Moreover, we show that any arbitrary $k$-firm merger becomes unprofitable if search costs and the number of non-merging firms are sufficiently high. These results establish a new merger paradox. What is interesting about this paradox is that it arises even if firms sell horizontally differentiated products and compete in prices.

Janssen and Moraga-González (2007) also study mergers in a consumer search market. In contrast with the present paper, they focus on markets where firms sell homogeneous products and the equilibrium is in mixed pricing strategies. Their main result is that mergers have redistributive effects with consumers searching little getting better off at the expense of consumers who search a lot. Since in the post-merger market consumers visit merging and non-merging firms in an order that maximizes expected utility, our paper is also related to the recent literature on ordered search. Arbatskaya (2007) studies a market for homogeneous products where the order in which firms are visited is exogenously given. In equilibrium 
prices must fall as the consumer walks away from the firms visited first. Zhou (2009a) considers the case of differentiated products and finds the opposite result. Armstrong, Vickers and Zhou (2009) study the implications of "prominence" in search markets. In their model, there is a firm that is always visited first and this firm charges lower prices and derives greater profits than the rest of the firms, which are visited randomly after consumers have visited the prominent firm. Zhou (2009b) extends the ideas in Armstrong et al. (2009) to the case in which a set of firms, rather than just one, is prominent. In our model, the merging stores, by raising their prices to internalize the pricing externalities they exert on one another, confer the non-merging firms a "prominent" position in the marketplace. In Haan and Moraga-González (2008), firms gain prominence by advertising more and/or better. They find that firms need not benefit from higher consumer search costs. Also related is the paper of Hortaçsu and Syverson (2004), who present a model where sampling probability variation across firms is used to explain price dispersion in the mutual funds industry.

The remainder of the paper is organized as follows. Section 2 describes the consumer search model. Section 3 presents the equilibrium analysis, for both cases, the pre-merger market and the post-merger market. Section 4 offers some concluding remarks. Various proofs are placed in an appendix to ease the reading of the paper.

\section{The model and the pre-merger symmetric equilibrium}

We use Wolinsky (1986) model of search for differentiated products. On the supply side of the market there are $n \geq 3$ firms selling horizontally differentiated products. All firms employ the same constant returns to scale technology of production and we normalize unit production costs to zero. On the demand side of the market, there is a unit mass of consumers. A consumer $m$ has tastes described by an indirect utility function

$$
u^{m i}\left(p_{i}\right)=\varepsilon_{m i}-p_{i},
$$

if she buys product $i$ at price $p_{i}$. The parameter $\varepsilon_{m i}$ can be thought of as a match value between consumer $m$ and product $i$. Match values are independently distributed across consumers and products. We assume

that the value $\varepsilon_{m i}$ is the realization of a random variable uniformly distributed on $[0,1]$. No firm can observe $\varepsilon_{m i}$ so practising price discrimination is not feasible. In what follows we will denote $z_{\ell} \equiv \max \left\{\varepsilon_{1}, \varepsilon_{2}, \ldots, \varepsilon_{\ell}\right\}$. For later reference, it will also be useful to calculate the optimal price of a multi-product monopolist selling $\ell$ varieties, which we denote $p_{\ell}^{m}$. This price maximizes $p\left(\operatorname{Pr}\left[z_{\ell} \geq p\right]\right)$, and this gives $p_{\ell}^{m}=(1+\ell)^{-\frac{1}{\ell}}$. Setting $\ell=1$, we have the single-product monopolist, whose price will simply be denoted $p^{m}$.

Consumers search sequentially with costless recall. We assume that search cost $s$ is relatively small so that the first search is always worth, that is:

$$
0 \leq s \leq \bar{s} \equiv \operatorname{Pr}\left[\varepsilon \geq p^{m}\right] E\left[\varepsilon-p^{m} \mid \varepsilon \geq p^{m}\right]
$$

which yields $\bar{s} \equiv\left(1-p^{m}\right)^{2} / 2$. When search cost is equal to zero, the model is similar to Perloff and Salop (1985). 


\section{Pre-merger market}

We focus on symmetric equilibrium. ${ }^{1}$ Following Wolinsky (1986), let $p^{*}$ denote the price charged by firms other than firm $i$ and consider the (expected) payoff to a firm $i$ which deviates from the symmetric equilibrium by charging a price $p_{i}$. Assume $p_{i} \geq p^{*}$ without loss of generality.

We start by computing the probability that a consumer accepts the offer of firm $i$, conditional on visiting firm $i$ first. Suppose that the purchase option at firm $i$ gives the buyer utility $\varepsilon_{i}-p_{i}$. If $\varepsilon_{i}-p_{i}<0$, the consumer will search again given our assumption $s<\bar{s}$. Suppose $\varepsilon_{i}-p_{i} \geq 0$. In equilibrium, a buyer who contemplates searching again expects to see a price of $p^{*}$ at the next shop to visit. Therefore, searching one more time, say at firm $j$, yields gains only if $\varepsilon_{j}>\varepsilon_{i}-p_{i}+p^{*} \equiv x$, i.e., if the consumer prefers the new option over option $i$. The expected benefit from searching once more is then

$$
\int_{x}^{1}(\varepsilon-x) d \varepsilon=\frac{1}{2}(1-x)^{2}
$$

Searching one more time is worthwhile if and only if these incremental benefits exceed the cost of search $s$. The buyer is exactly indifferent between searching once more and stopping and accepting the offer at hand if $x=\bar{x}$, with $\bar{x}$ given by the solution to $\frac{1}{2}(1-x)^{2}=s$, i.e., $\bar{x}=1-\sqrt{2 s}$. Since $s \in\left[0,\left(1-p_{\ell}^{m}\right)^{2} / 2\right]$, we have that $\bar{x} \in\left[p_{\ell}^{m}, 1\right]$.

In any equilibrium $\bar{x} \geq p^{*}$. Therefore, the probability that a buyer stops searching at firm $i$ given that firm $i$ is visited first, is equal to

$$
\operatorname{Pr}[x>\bar{x}]=1-\bar{x}-p_{i}+p^{*}
$$

provided the deviating price is not too high, i.e., $p_{i}<1-\bar{x}+p^{*}$ for otherwise every single consumer would walk away from firm $i .^{2}$

The consumer may visit firm $i$ after having visited other firm(s). The probability that a consumer goes to firm $i$ in her second search and decides to acquire the offering of firm $i$ right away is $\bar{x}\left(1-\bar{x}-p_{i}+p^{*}\right){ }^{3}$ Similarly, the probability that a consumer goes to firm $i$ in her $\ell$-th search and decides to acquire the offering of firm $i$ right away is $\bar{x}^{\ell-1}\left(1-\bar{x}-p_{i}+p^{*}\right)$.

To complete firm $i$ 's payoff calculation, we need to compute the joint probability that a consumer walks away from every single firm in the market and happens to return to firm $i$ to conduct a transaction, that is

$$
\operatorname{Pr}\left[\max \left\{0, z_{n-1}-p^{*}\right\}<\varepsilon_{i}-p_{i}<\bar{x}-p^{*}\right]
$$

\footnotetext{
${ }^{1}$ We note that asymmetric equilibria can be sustained in this model. The idea is that if consumers believe that firms' prices are, say, ordered as follows $p_{1}<p_{2}<\ldots<p_{n}$, then it is optimal for consumers to visit firms in that order and for firms to price in a way to make consumer beliefs coherent. The unattractive feature of these equilibria is that they are not determined by the underlying characteristics of the market, but by an indeterminacy of beliefs. We will ignore this types of equilibria in our paper. A completely different situation is that studied in Zhou (2011) where it is assumed that the shops of the firms are arranged in a particular way so consumers have no alternative than to visit them in a pre-specified exogenous order.

${ }^{2}$ In what follows we derive the payoff of a firm under the assumption that $p_{i}<1-\bar{x}+p^{*}$. When this does not hold, the payoff is slightly different. We deal with this case later (see footnote 4 ).

${ }^{3}$ Letting $j$ denote the firm visited earlier, this probability is given by $\operatorname{Pr}\left[\varepsilon_{i}-p_{i}>\bar{x}-p^{*}>\varepsilon_{j}-p^{*}\right]$.
} 
This probability is independent of the order in which firms are visited. We will denote it as $r_{a}$ to indicate that these are consumers who return to a firm $i$ after having visited all the firms in the market. We then have:

$$
r_{a} \equiv \int_{p_{i}}^{\bar{x}+p_{i}-p^{*}}\left(\varepsilon_{i}-p_{i}+p^{*}\right)^{n-1} d \varepsilon_{i}=\int_{0}^{\bar{x}-p^{*}}\left(\varepsilon_{i}+p^{*}\right)^{n-1} d \varepsilon_{i}=\frac{1}{n}\left(\bar{x}^{n}-p^{* n}\right) .
$$

Using the notation introduced above, we can now write firm $i$ 's expected profits:

$$
\pi_{i}=p_{i}\left[\frac{1-\bar{x}^{n}}{n(1-\bar{x})}\left(1-\bar{x}-p_{i}+p^{*}\right)+r_{a}\right] .
$$

We look for a symmetric Nash equilibrium in prices. After applying symmetry and the requirement that consumer expectations are fulfilled, i.e., $p_{i}=p^{*}$, the first-order condition (FOC) is:

$$
1-p^{* n}-p^{*} \frac{1-\bar{x}^{n}}{1-\bar{x}}=0
$$

It is easy to check that (4) has a unique solution that satisfies $\bar{x} \geq p^{*} \geq 1-\bar{x}^{4}$ In addition, one can show that the equilibrium price increases in the search cost $s$ (Wolinsky, 1986; Anderson and Renault, 1999).

The profits of a typical firm in the pre-merger situation are

$$
\pi^{*}=\frac{1}{n} p^{*}\left(1-p^{* n}\right) .
$$

\section{Equilibrium when $k$ firms merge}

In this section we study the price implications of mergers and the incentives to merge. As in Deneckere and Davidson (1985), we abstract from efficiency gains and focus on the effects of joint (price) decisionmaking. Consider that $k$ firms merge, with $2 \leq k \leq n-1$. In what follows, a typical merging firm will be denoted $i$, while a typical non-merging store will be denoted $j$.

As before we focus on symmetric equilibria in the sense that all non-merging firms will be assumed to charge $\tilde{p}^{*}$, and all merging firms will be supposed to charge $\hat{p}^{*}$. As it is expected, suppose also that the merging firms charge higher prices than the non-merging firms, i.e., $\tilde{p}^{*}<\hat{p}^{*}$. This is reasonable because internalizing the pricing externalities the merging firms confer on one another lead these firms to charge higher prices than the non-merging ones. ${ }^{5}$

\footnotetext{
${ }^{4}$ The equilibrium price $p^{*}$ is indeed an equilibrium if no firm has an incentive to deviate from it. So far we have checked that "small" deviations are not profitable. Suppose now that the deviant firm charges a price so high that consumers always walk away from it and therefore this firm only sells to those consumers who come back to it after having visited all other firms. In that case the deviant profits become $\Pi_{i}\left(p_{i} ; p^{*}\right)=p_{i} \int_{p_{i}}^{1}\left(\varepsilon_{i}-p_{i}+p^{*}\right)^{n-1} d \varepsilon_{i}$. Because of log-concavity of the uniform density function, this profits expression is quasi-concave in own price (Caplin and Nalebuff, 1991). Taking the derivative of the deviating profits with respect to $p_{i}$, and setting $p_{i}=p^{*}$, we get $d \Pi_{i} /\left.d p_{i}\right|_{p_{i}=p^{*}}=\left(1-p^{* n}-n p^{*}\right) / n<0$, where the inequality follows from the fact that $p^{*}$ solves (4). Since deviating profits are quasi-concave and they decrease at $p_{i}=p^{*}$, we conclude they are even lower at prices $p_{i}$ such that $\bar{x}+p_{i}-p^{*}>1$.

${ }^{5} \mathrm{~A}$ comment on the existence of other equilibria is in order now. As in the pre-merger market, it may be possible to sustain asymmetric equilibria in the sense that distinct non-merging and/or distinct merging firms charge different prices. Again, these asymmetries are not based on any underlying characteristic of the market and can only be sustained because of the indeterminacy of consumer beliefs discussed in the previous section. We will abstract from these types of asymmetric
} 
Given this, optimal consumer search (see e.g. Kohn and Shavell, 1974) implies consumers should start searching for a satisfactory product at the non-merging firms and then, if no alternative is found to be good enough in those firms, continue searching at the merging ones. To calculate the equilibrium, we proceed by computing the payoff the two types of stores (merging and non-merging) would obtain when deviating from the equilibrium prices. Then we derive the FOCs, impose the symmetry of prices across firms of the same type, require consumer expectations to be correct, and solve for equilibrium prices.

\section{Payoff to a deviant non-merging store.}

We now compute the payoff of a non-merging store $j$ that deviates from $\tilde{p}^{*}$ by charging $\tilde{p} \neq \tilde{p}$. As all non-merging firms are supposed to charge the same price $\tilde{p}^{*}$, consumers are assumed to visit them randomly. Therefore the deviant firm may be visited in first place, second place and so on till the $(n-k)$-th place. As any other non-merging store, the deviant has a probability $1 /(n-k)$ of being visited in each of these positions. When the consumer visits the deviant in the 1st, $2 \mathrm{nd}, \ldots,(n-k-1)$-th place, the decision whether to continue searching or not takes into account that the next visited shop is also a non-merging store. By contrast, when the deviant firm is the last non-merging store visited by the consumer, i.e. the $(n-k)$-th, the decision of the consumer is slightly different because the next shop to be visited is a merging store and such a store charges a price different from the price of a non-merging store. Since the consumer stopping rule is different at any of the first $n-k-1$ non-merging stores than that at the last non-merging store, it is convenient to distinguish among those two cases.

- Consider the deviant non-merging firm $j$ that is visited by a consumer in $h$-th place, with $h=$ $1,2, \ldots, n-k-1$. Suppose the deal a consumer observes upon entering the deviant's shop is $\varepsilon_{j}-\tilde{p}$. There are three circumstances in which the deviant sells to this consumer.

- First, the consumer may stop searching at this shop and buy there right away. This occurs when $\varepsilon_{j} \geq \bar{x}-\tilde{p}^{*}+\tilde{p}$, where $\bar{x}$ was defined in section 2 . Therefore, the joint probability a consumer visits the deviant in $h$-th place and buys there directly is

$$
\operatorname{Pr}\left[z_{h-1}-\tilde{p}^{*}<\bar{x}-\tilde{p}^{*}<\varepsilon_{j}-\tilde{p}\right]=\bar{x}^{h-1}\left(1-\bar{x}+\tilde{p}^{*}-\tilde{p}\right)
$$

- Second, the consumer may walk away from the firm visited in $h$-th place and come back to it after visiting all non-merging stores. To see this, note that optimal search implies that the

equilibria. What may also happen is that a symmetric equilibrium where the merging firms charge a price lower than the non-merging firms, i.e. $\tilde{p}^{*}>\hat{p}^{*}$, exists. This equilibrium is counterintuitive because we know that joint-profit maximization leads the merging firms to internalize the pricing externalities they impose on one another, which calls for higher rather than lower prices than the non-merging firms. Therefore, if this type of symmetric equilibrium exists, it must be because the force of consumer beliefs more than offsets the effect of joint profit-maximization. Later in Section 4.2 we prove that an equilibrium with $\tilde{p}^{*}>\hat{p}^{*}$ fails to exist when for example the search cost is low or when the search cost is high. In those cases, the strength of the order effect driven by consumer beliefs is relatively weak. The implication of this result is that if one takes seriously such an equilibrium where the merging firms charge lower prices than the non-merging ones, consumer beliefs should be discontinuous in search costs, which is difficult to justify. In Section 4.2, we also show that this type of equilibrium can easily be destabilized when for example there exist consumers in the market who have zero search costs. 
consumer would walk away from the last non-merging store to visit one of the merging firms if $z_{n-k} \leq \bar{x}-\hat{p}^{*}+\tilde{p}^{*}$. Moreover, if the consumer did arrive to the $(n-k)$-th non-merging store it is because $z_{n-k-1} \leq \bar{x}$. Since $\hat{p}^{*}>\tilde{p}^{*}$, it is clear that the condition to leave the last non-merging store and continue searching among the merging stores is more stringent than that to continue searching among the non-merging stores. For this reason, the consumer may return to the deviant firm after having visited all non-merging firms and buy there. This occurs when

$$
\operatorname{Pr}\left[\max \left\{z_{n-k-1}-\tilde{p}^{*}, \bar{x}-\hat{p}^{*}\right\}<\tilde{\varepsilon}_{j}-\tilde{p}<\bar{x}-\tilde{p}^{*}\right]
$$

and this gives the following"coming back"or"returning"demand:

$$
\tilde{r}_{n m} \equiv \int_{\bar{x}-\hat{p}^{*}+\tilde{p}}^{\bar{x}-\tilde{p}^{*}+\tilde{p}}\left(\varepsilon_{j}-\tilde{p}+\tilde{p}^{*}\right)^{n-k-1} d \varepsilon_{j}=\frac{1}{n-k}\left(\bar{x}^{n-k}-\left(\bar{x}-\hat{p}^{*}+\tilde{p}^{*}\right)^{n-k}\right)
$$

where the subindex " $n m$ " refers to the fact that consumers return to the deviant firm after having visited all the non-merging stores.

- Finally, the consumer may walk away from the deviant non-merging firm and come back to it after having visited all the firms in the market. This occurs when

$$
\operatorname{Pr}\left[\max \left\{z_{n-k-1}-\tilde{p}^{*}, z_{k}-\hat{p}^{*}, 0\right\}<\tilde{\varepsilon}_{j}-\tilde{p}<\bar{x}-\hat{p}^{*}\right]
$$

and this gives the following "coming-back" demand

$$
\tilde{r}_{a} \equiv \int_{0}^{\bar{x}-\hat{p}^{*}}\left(\varepsilon_{j}+\tilde{p}^{*}\right)^{n-k-1}\left(\varepsilon_{j}+\hat{p}^{*}\right)^{k} d \varepsilon_{j}
$$

where the subindex " $a$ " again refers to the fact that consumers return after having visited all the stores.

- We now consider the case in which the deviant firm is visited in $(n-k)$-th place. This type of firm sells to the consumers in two cases:

- First, the consumer stops searching at this shop and buys there right away. This occurs with probability

$$
\operatorname{Pr}\left[\varepsilon_{j}-\tilde{p} \geq \max \left\{z_{n-k-1}-\tilde{p}^{*}, \bar{x}-\hat{p}^{*}\right\} \text { and } z_{n-k-1}<\bar{x}\right]
$$

and this gives a demand

$$
\bar{x}^{n-k-1}\left(1-\bar{x}+\tilde{p}^{*}-\tilde{p}\right)+\frac{1}{n-k}\left[\bar{x}^{n-k}-\left(\bar{x}-\hat{p}^{*}+\tilde{p}^{*}\right)^{n-k}\right]
$$

- Second, the consumer walks away from this firm and comes back to it after visiting all the firms in the market. In this second case we have exactly the same expression for returning consumers as in (6). 
As a result, taking into account the different positions in which the deviant firm may be visited by a consumer, we get the profits of a deviant non-merging firm:

$$
\tilde{\pi}=\tilde{p}\left[\frac{1}{n-k} \frac{1-\bar{x}^{n-k}}{1-\bar{x}}\left(1-\bar{x}+\tilde{p}^{*}-\tilde{p}\right)+\tilde{r}_{n m}+\tilde{r}_{a}\right]
$$

\section{Payoff to a deviant merging store.}

We now compute the joint payoff of the merging stores. Recall that since consumers expect the price set at the merging firms $\hat{p}^{*}$ to be greater than $\tilde{p}^{*}$, they postpone visiting them until they have visited all the non-merging firms. Suppose that the merging firms deviate by charging $\hat{p} \neq \hat{p}^{*}$.

Consider a consumer who walks away form the last non-merging store and observes a deal $\varepsilon_{i}-\hat{p}$ at the first merging store she visits. We note first that such a consumer will never return to any of the non-merged firms without first visiting all other merging stores. This is because the utilities from all non-merged firms are lower than $\bar{x}-\hat{p}^{*}$, which is exactly the reservation utility at any of the merging shops. We now ask whether the consumer will continue searching after she visits the first merging shop. Clearly, she will continue searching when her best deal so far does not give her positive utility. She will do the same when the highest utility so far is obtained at one of the non-merging stores, that is, $z_{n-k}-\tilde{p}^{*}>\varepsilon_{i}-\hat{p}>0$. In case the best deal is the one at the merging store, the consumer will continue searching when $\varepsilon_{i}-\hat{p}<\bar{x}-\hat{p}^{*}{ }^{6}$ As a result, the probability that the consumer arrives at the first merging store and buys there right away is

$$
\operatorname{Pr}\left[\varepsilon_{i}-\hat{p} \geq \bar{x}-\hat{p}^{*} \geq \max \left\{z_{n-k}-\tilde{p}^{*}\right]=\left(\bar{x}-\hat{p}^{*}+\tilde{p}^{*}\right)^{n-k}\left(1-\bar{x}+\hat{p}^{*}-\hat{p}\right)\right.
$$

Suppose now the consumer walks into the $h$-th merged store, $h=2, \ldots, k$. The probability this consumer buys at that shop right away is

$$
\operatorname{Pr}\left[\max \left\{z_{h-1}-\hat{p}, z_{n-k}-\tilde{p}^{*}\right\}<\bar{x}-\hat{p}^{*}<\varepsilon_{i}-\hat{p}\right]
$$

This gives a demand $\left(\bar{x}-\hat{p}^{*}+\tilde{p}^{*}\right)^{n-k}\left(\bar{x}-\hat{p}^{*}+\hat{p}\right)^{h-1}\left(1-\bar{x}+\hat{p}^{*}-\hat{p}\right)$. Taking into account the different positions in which a merging firm may be visited, we have a demand equal to

$$
\left(\bar{x}-\hat{p}^{*}+\tilde{p}^{*}\right)^{n-k} \sum_{h=1}^{k}\left(\bar{x}-\hat{p}^{*}+\hat{p}\right)^{h-1}\left(1-\bar{x}+\hat{p}^{*}-\hat{p}\right)=\left(\bar{x}-\hat{p}^{*}+\tilde{p}^{*}\right)^{n-k}\left[1-\left(\bar{x}-\hat{p}^{*}+\hat{p}\right)^{k}\right]
$$

Some consumers visit all shops in the market and decide to return to one of the merging stores to conduct a purchase. Then, the joint returning demand obtained by the merging stores is given by:

$$
\sum_{h=1}^{k} \operatorname{Pr}\left[\varepsilon_{i} \geq \max \left\{z_{k-1}, z_{n-k}+\hat{p}-\tilde{p}^{*}, \hat{p}\right\} \text { and } \varepsilon_{i}<\bar{x}-\hat{p}^{*}+\hat{p}\right]
$$

\footnotetext{
${ }^{6}$ We assume that a buyer who observes a deviation price $\hat{p}$ at one of the merging stores does not change the expectation that the other merged firms charge $\hat{p}^{*}$. This assumption is adopted for technical reasons though it can easily be justified because consumers usually need not know both whether the pricing of firms is joint or independent, or the ownership structure of the firms. If consumers did know the merging firm deviates jointly in all its stores, then they would update their expectations correspondingly and this would lead to a kink in the demand function of the merging firms. In this situation, downward deviations lead to exactly the same payoff as here. However, upward deviations lead to a different payoff function, which suggests the existence of multiple equilibria. Details can be obtained from the authors upon request.
} 
which gives the following "coming-back" demand

$$
\hat{r}_{a} \equiv k \int_{0}^{\bar{x}-\hat{p}^{*}}\left(\varepsilon_{i}+\tilde{p}^{*}\right)^{n-k}\left(\varepsilon_{i}+\hat{p}\right)^{k-1} d \varepsilon_{i}
$$

The payoff to a deviating merged entity is then:

$$
\hat{\pi}=\hat{p}\left[\left(\bar{x}-\hat{p}^{*}+\tilde{p}^{*}\right)^{n-k}\left(1-\left(\bar{x}-\hat{p}^{*}+\hat{p}\right)^{k}\right)+\hat{r}_{a}\right]
$$

\subsection{An example with three firms}

In order to develop some intuition for the main results, we briefly discuss in this subsection an example with three firms in which two of them merge. Prices and profits in the pre-merger market follow straightforwardly from setting $n=3$ in (4) and (5).

Firms payoffs in the post-merger market are

$$
\begin{gathered}
\tilde{\pi}=\tilde{p}\left[1-\bar{x}-\tilde{p}+\hat{p}^{*}+\frac{1}{3}\left(\bar{x}^{3}-\hat{p}^{* 3}\right)\right] \\
\hat{\pi}=\hat{p}\left[\left(\bar{x}-\hat{p}^{*}+\tilde{p}^{*}\right)\left(1-\left(\bar{x}-\hat{p}^{*}+\hat{p}\right)^{2}\right)+2 \int_{\hat{p}}^{\bar{x}-\hat{p}^{*}+\hat{p}} \varepsilon\left(\varepsilon-\hat{p}^{*}+\tilde{p}^{*}\right) d \varepsilon\right]
\end{gathered}
$$

with corresponding FOCs after applying symmetry

$$
\begin{array}{r}
1-2 \tilde{p}^{*}+\hat{p}^{*}-\bar{x}+\frac{1}{3}\left(\bar{x}^{3}-\hat{p}^{* 3}\right)=0 \\
\frac{4 \hat{p}^{* 3}}{3}+\tilde{p}^{*}-3 \hat{p}^{* 2} \tilde{p}^{*}+\bar{x}-\frac{\bar{x}^{3}}{3}-\hat{p}^{*}\left(1+\bar{x}^{2}\right)=0
\end{array}
$$

It can be seen that this system of equations has always a solution and that such solution is unique and constitutes a Nash equilibrium. ${ }^{7}$ For a fixed search cost, it is straightforward to solve the FOCs numerically for equilibrium prices. The results are given in Figure 1a, where we plot post-merger equilibrium prices, $\hat{p}^{*}$ and $\tilde{p}^{*}$, against search costs. For comparison purposes, we also plot the pre-merger price, $p^{*}$. As expected, all prices are increasing in search costs. As searching for price and product fit becomes more costly, firms have more market power over the consumers who pay them a visit and this results in higher prices for all the firms.

As the graph reveals, post-merger prices, whether from merging or non-merging firms, happen to be higher than the pre-merger price. This deserves a comment. In our model, the non-merging firm is expected to charge a price lower than that of the merging stores and therefore it is visited first by the consumers; after that, if consumers do not find a satisfactory product there, they proceed by checking the goods on sale at the merging stores. In the terminology of Armstrong et al. (2009), what happens in our model is that by merging, the joining firms end up conferring market prominence to the non-merging store.

Armstrong et al. (2009) study the effects of market prominence. They show, on the one hand, that a prominent firm charges a lower price than the rest of the firms, as it is here the case. In their model this price ranking originates from the order in which firms are visited by consumers. The firm that is visited

\footnotetext{
${ }^{7}$ Further, we can show that no other symmetric equilibrium exists (see Proposition ??(c).
} 


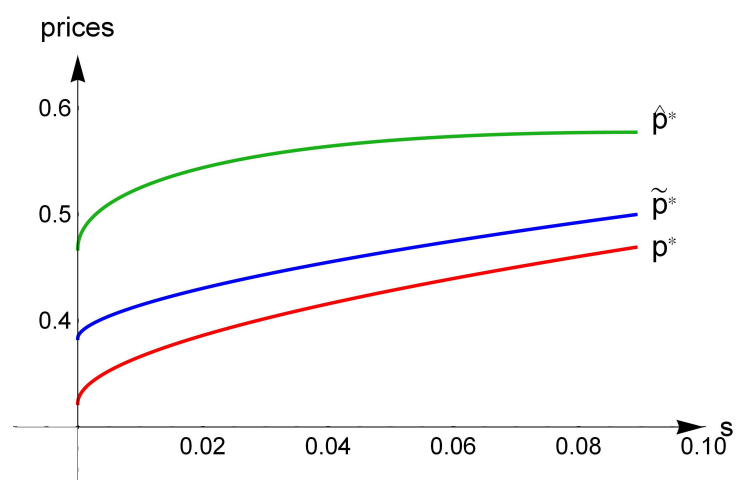

(a) Prices

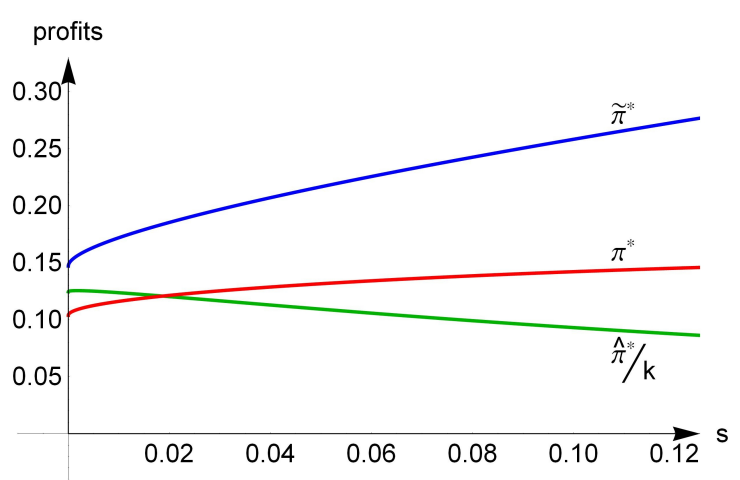

(b) Profits

Figure 1: Pre- and post-merger prices, and merger profitability.

first has a more elastic demand than the other firms just because the latter receive consumers who were dissatisfied at the first firm so in effect it is as if they had fewer acceptable options. In addition, Armstrong et al. show that the prominent firm charges a lower price than in the case in which no prominent firm exists. This result does not arise here. The reason is that in our model there is a second force counteracting with the search-order effect: merging firms internalize pricing externalities between them and raise prices over and above the prices they would charge if they were operating independent stores. This weakens competition further and then all prices increase over and above the status quo (pre-merger) situation.

Figure $1 \mathrm{~b}$ shows how the profits of a merging firm and a non-merging firm, $\hat{\pi}^{*} / k$ and $\tilde{\pi}^{*}$, vary with search costs. In addition, the figure gives the pre-merger profits, $\pi^{*}$, so we can readily assess whether merging is worthwhile for the merging parties. The most important point to make here is that the profits of a merging firm decline as search cost goes up. The reason is that, as the search cost increases, fewer consumers walk away from the non-merging firm and visit the merging firms. This has a major implication on merger profitability: for sufficiently large search costs, merging is not individually rational for the merging entities. The graph reveals that the non-merging firm gets a free ride and this ride is freer the higher the search cost. This result resembles the well-known merger paradox, but its novelty is that it arises under price competition with differentiated product sellers. These insights can be proven more generally and this will be the purpose of the next section and the Appendix.

\subsection{Main results}

We first study the existence of equilibrium. For this, we first define a critical search cost value below which all firms, including the merging firms, receive positive demand. Let

$$
\tilde{s}_{k} \equiv \operatorname{Pr}\left[\varepsilon \geq p_{k}^{m}\right] E\left[\varepsilon-p_{k}^{m} \mid \varepsilon \geq p_{k}^{m}\right] .
$$

$\tilde{s}_{k}$ is the search cost that makes a consumer who has currently found no acceptable option indifferent between staying at home and visiting a monopoly firm that controls $k$ stores. Since $p_{k}^{m}=(1+k)^{-\frac{1}{k}}$, $\tilde{s}_{k}=\left(1-(1+k)^{-\frac{1}{k}}\right)^{2} / 2$. In what follows, if a merger of $k$ firms occurs, we will pay only attention to search 
costs in the set $\left[0, \tilde{s}_{k}\right]$ (or $\left.\bar{x} \in\left[p_{k}^{m}, 1\right]\right)$. This ensures that each of the $k$ merging stores has positive demand.

Taking the first order derivatives of the payoffs in (7) and (9) with respect to deviation prices $\hat{p}$ and $\tilde{p}$ respectively and applying the equilibrium requirement that consumer beliefs are correct, i.e., $\hat{p}^{*}=\hat{p}$ and $\tilde{p}^{*}=\tilde{p}$, yields the following FOCs:

$$
\begin{gathered}
\left(\bar{x}-\hat{p}^{*}+\tilde{p}^{*}\right)^{n-k}\left(1-\bar{x}^{k}-k \hat{p}^{*} \bar{x}^{k-1}\right)+k \int_{0}^{\bar{x}-\hat{p}^{*}}\left(\varepsilon+\tilde{p}^{*}\right)^{n-k}\left(\varepsilon+\hat{p}^{*}\right)^{k-2}\left(\varepsilon+k \hat{p}^{*}\right) d \varepsilon=0 \\
1-\frac{1-\bar{x}^{n-k}}{1-\bar{x}} \tilde{p}^{*}-\left(\bar{x}-\hat{p}^{*}+\tilde{p}^{*}\right)^{n-k}+(n-k) \int_{0}^{\bar{x}-\hat{p}^{*}}\left(\varepsilon+\tilde{p}^{*}\right)^{n-k-1}\left(\varepsilon+\hat{p}^{*}\right)^{k} d \varepsilon=0
\end{gathered}
$$

Proposition 1. Assume that $k$ firms merge. For any $s \in\left[0, \tilde{s}_{k}\right]$, there exists a Nash equilibrium in the post-merger market where consumers start searching at the non-merging stores and then they proceed by searching at the merged ones. Merging firms charge a price $\hat{p}^{*}$ and the non-merging stores charge a price $\tilde{p}^{*} ;$ these prices are given by the unique solution to the system of FOCs (12)-(13) and the price ranking is consistent with consumer search behavior, that is, $\hat{p}^{*}>\tilde{p}^{*}$.

The proof of this Proposition, which is presented in the Appendix, has the following steps. We first show that there is a unique pair of prices $\left\{\hat{p}^{*}, \tilde{p}^{*}\right\}$ that satisfies the FOCs. We then show that these prices satisfy the inequality $\hat{p}^{*}>\tilde{p}^{*}$, which immediately implies that the hypothesized consumer search behavior is optimal.

Proposition 1 follows from the behavior of the reaction functions of the different types of firm. We illustrate these ideas using Figure 2. In this Figure, the crossing point between the two blue reaction functions gives the pre-merger equilibrium. The joint reaction function of the potentially merging firms is denoted by $r_{k}^{p r e}$, while the joint reaction function of the non-merging firms is denoted by $r_{n-k}^{\text {pre }}$. In the pre-merger market, consumers expect all firms to charge the same price so they visit firms randomly. The joint reaction functions cross on the 45 degrees line so both types of firms charge $p^{*}$ and consumers' expectations are fulfilled.

When the potentially merging firms do indeed merge, two effects take place. On the one hand, there is a search-order effect. Since the insider firms are supposed to internalize pricing externalities and therefore raise their prices more than the outsider firms, consumers push the merging firms all the way back in the queue they follow when they search for satisfactory products. On the other hand, there is an internalization-of-pricing-externalities effect, as usual when firms merge.

By the search-order effect, the joint reaction curve of the merging firms shifts upwards from $r_{k}^{p r e}$ (in blue) to $r_{k}$ (in green). This move captures the fact that the merging firms' demand becomes more inelastic just because only consumers who have been disappointed at the non-merging stores happen to reach the merged stores. By the same token, the joint reaction curve of the non-merging firms also changes because of this search-order effect. In particular, it shifts to the left and turns clockwise from $r_{n-k}^{\text {pre }}$ (in blue) to $r_{n-k}^{\text {post }}$ (in red). This move is the result of these firms gaining "prominence" in the marketplace. The crossing point between the curves $r_{k}$ and $r_{n-k}^{\text {post }}$ gives the Nash equilibrium of the pricing game when $n-k$ firms are visited first and the rest $k$ of the firms later. This situation has been studied by Armstrong et al. 
(2009) and Zhou (2009). When there is just one prominent firm, Armstrong et al. (2009) show that the price of the prominent firm will be lower than the price the firms would charge in a symmetric situation. Zhou (2009) qualifies this result demonstrating that when the number of prominent firms in the market is more than 1, the price of prominent firms can be higher than the price firms would charge if they were all symmetric.

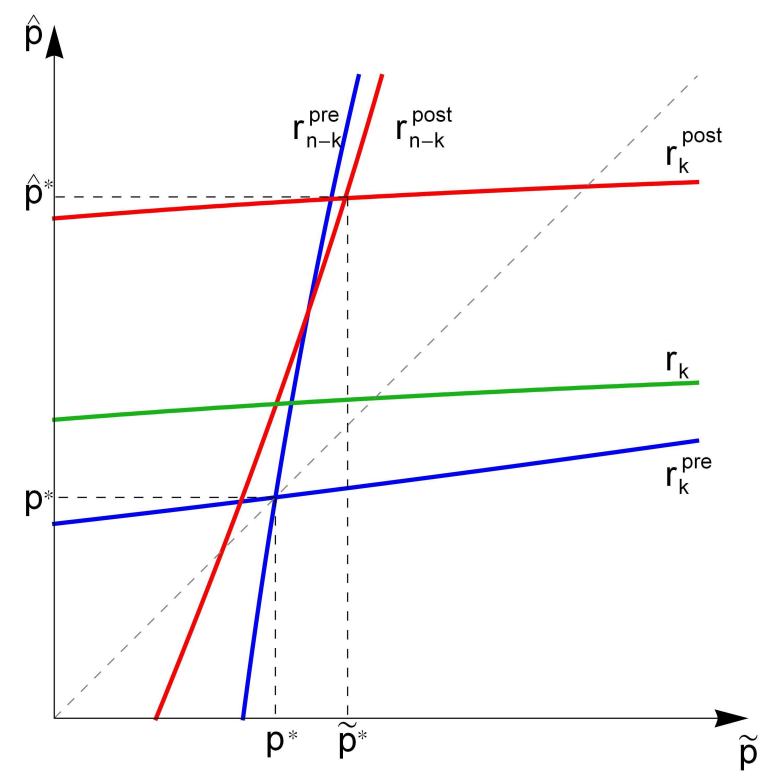

Figure 2: Post-merger and pre-merger equilibria.

The change in the joint reaction curve of the merging stores due to the search-order effect is augmented by the usual internalization-of-pricing-externalities effect, which shifts the joint reaction function of the merging stores further from $r_{k}$ (in green) to $r_{k}^{\text {post }}$ (in red). By strategic complementarity, the shift in the reaction curve of the merging stores pushes all prices, whether from outsiders or insiders, up. The post-merger equilibrium is given by the crossing point of the two red curves.

We explore next the relationship between the post-merger equilibrium prices and the pre-merger equilibrium price.

Proposition 2. The ranking of pre-and post-merger equilibrium prices is $p^{*}<\tilde{p}^{*}<\hat{p}^{*}$ whenever one of the following conditions holds: (a) the search cost is sufficiently low, (b) the search cost is sufficiently high, (c) the number of firms $n=3$.

From the discussion above around Figure 2, it should be clear that whether the post-merger equilibrium prices are higher or lower than the pre-merger equilibrium price is a priori ambiguous. Consider the price charged by the non-merging firms. The fact that the potentially merging stores by actually merging confer a "prominent" position in the marketplace to the non-merging stores causes a direct downward pressure on the price of the non-merging firms. As said before, when a firm becomes prominent its pool of consumers becomes more elastic. In addition there are two indirect effects. The first is that, since the merging firms are relegated to the last positions of the queue consumers follow when they search, they 
tend to raise their prices. This effect arises because for the non-prominent firms it holds the opposite, namely, that their demand becomes less elastic. By strategic complementarity, this weakens competition in the marketplace and the non-merging firms tend to raise their prices as well. The last effect follows from the fact that the merging firms internalize the pricing externalities they confer on one another. This also tends to raise their price, and indirectly, by strategic complementarity again, the prices of the non-merging firms. Similar considerations apply to the price of the merging firms. Our proposition shows that when the search-order effects are not very strong then we are sure that all prices increase after a merger. We note however that solving numerically the model we have found no example in which this does not happen. Basically, what we always observe is similar to what happens in Figure 1a when $n=3$.

We study next the relationship between the post-merger equilibrium prices and search costs. The following result extends those in Wolinsky (1986) and Anderson and Renault (1999) about how search costs influence the symmetric equilibrium price to the merger situation studied here.

Proposition 3. The post-merger equilibrium prices $\hat{p}^{*}$ and $\tilde{p}^{*}$ increase in search costs.

Our final result explores merger profitability.

Proposition 4. Assume that search cost $s \in\left[0, \tilde{s}_{k}\right]$. Then: (a) Any 2-firm merger is not profitable if the search cost is sufficiently high. (b) Any arbitrary $k$-firm merger is not profitable if the search cost and the number of competitors are sufficiently high. (c) If search costs are sufficiently small, any arbitrary $k$-firm merger is profitable.

As expected, the case in which search cost is small reproduces naturally the situation in Deneckere and Davidson (1985). However, as search costs increase, fewer consumers walk away from the non-merging stores and visit the merged ones. This lowers the profits of the merging firms. Eventually, when the search cost becomes relatively high, unless there are many firms in the industry and the merger comprises almost all of them, merging becomes unprofitable. The interest of this Proposition is that it puts forward a new merger paradox, which arises under price competition with differentiated products. The underlying reason is based on search costs, something quite different from the merger paradox of Salant et al. (1983), which concerns competition with decision variables that are strategic substitutes.

\section{Discussion}

\subsection{Alternative distributions of consumer tastes}

So far we have assumed that consumer tastes are uniformly distributed on the unit interval. In this section we study the sensitiveness of our results to this assumption. For this purpose, we assume that the distribution of match values is $F(\varepsilon)=\varepsilon^{\lambda}$ with $\varepsilon \in[0,1]$ and $\lambda \geq 1$. The parameter $\lambda$ is a shifter of the distribution of consumer preferences towards the right end of the interval of possible match values. When $\lambda$ goes up, products become more homogeneous and, therefore, competition between firms becomes fiercer. Note also that the average willingness to pay, which is $\lambda /(1+\lambda)$, also increases in the parameter $\lambda$.

With this distribution of match values, the price of a monopolist selling $\ell$ varieties becomes $p_{\ell}^{m}=$ $(1+\lambda \ell)^{-1 / \lambda \ell}$. The threshold match value $\tilde{x}$ above which consumers stop searching becomes the solution to 
$\int_{\tilde{x}}^{1}(\varepsilon-\tilde{x}) d \varepsilon^{\lambda}=s$. This gives a critical search cost $\tilde{s}_{\ell}=\frac{\lambda}{1+\lambda}-p_{\ell}^{m}+\frac{1}{1+\lambda}\left(p_{\ell}^{m}\right)^{\lambda+1}$ beyond which no consumer would ever visit the shops of a monopoly firm in control of $\ell$ shops. One can easily check that when $\lambda=1$ we get the same expressions for the monopoly price and maximum search cost we had in section 2 .

For simplicity, let us assume that $n=3$, as in section 3 . It is easy to rewrite the payoffs of the firms using the new distribution of match values. These are

$$
\begin{aligned}
& \tilde{\pi}\left(\tilde{p}, \hat{p}^{*}\right)=\tilde{p}\left[1-\left(\tilde{x}+\tilde{p}-\hat{p}^{*}\right)^{\lambda}+\int_{\tilde{p}}^{\tilde{x}-\hat{p}^{*}+\tilde{p}} \lambda \varepsilon^{\lambda-1}\left(\varepsilon-\tilde{p}+\hat{p}^{*}\right)^{2 \lambda} d \varepsilon\right] \\
& \hat{\pi}\left(\tilde{p}^{*}, \hat{p}\right)=\hat{p}\left[\left(1-\left(\tilde{x}-\hat{p}^{*}+\hat{p}\right)^{2 \lambda}\right)\left(\tilde{x}+\tilde{p}^{*}-\hat{p}^{*}\right)^{\lambda}+2 \int_{\hat{p}}^{\bar{x}-\hat{p}^{*}+\hat{p}} \lambda \varepsilon^{2 \lambda-1}\left(\varepsilon-\hat{p}+\tilde{p}^{*}\right)^{\lambda} d \varepsilon\right]
\end{aligned}
$$

As above, equilibrium prices can be found by taking the FOCs, setting $\hat{p}=\hat{p}^{*}$ and $\tilde{p}=\tilde{p}^{*}$ and solving for $\hat{p}^{*}$ and $\tilde{p}^{*}$. Even in this simple environment with three firms, calculations get complicated when $\lambda$ can vary freely.

Figure 3a shows the pre-merger and post-merger equilibrium prices of Proposition 1. Initially there are three firms and then two of them merge. The search cost is fixed at $s=0.01$ and $\lambda$ varies from 1 to 10 . The graph shows that no matter the value of $\lambda$, the price of the non-merging firms is lower than the price of the merging firms. Moreover, we see that as $\lambda$ goes up, prices increase and then decrease. To understand this, recall that as $\lambda$ goes up the intensity of competition increases and this tends to lower prices. At the same time, as $\lambda$ goes up, the average willingness-to-pay also increases and this pushes prices up. The first effect dominates for high levels of $\lambda$, while it happens otherwise for low levels of $\lambda$.

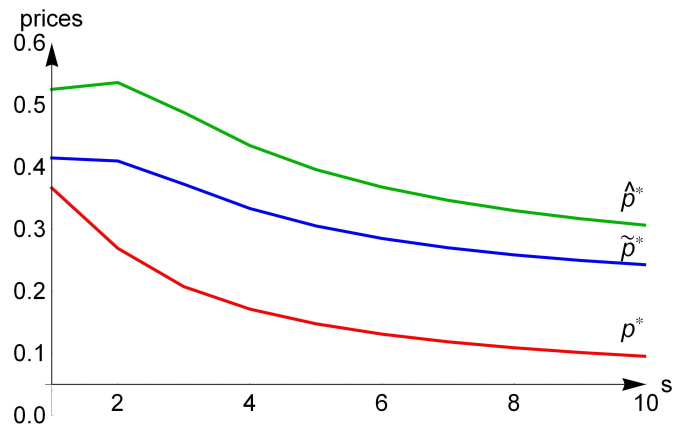

(a) Prices

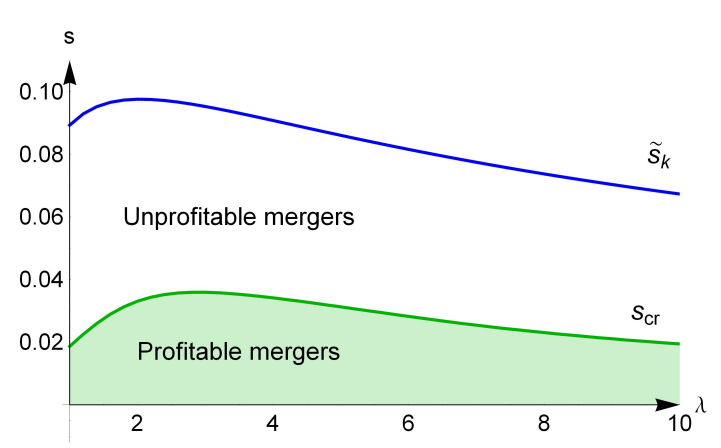

(b) Merger profitability

Figure 3: Pre- and post-merger prices and merger profitability.

In Figure $3 \mathrm{~b}$ we show the region of search costs $s$ and taste parameters $\lambda$ for which merging is individually rational. The critical search cost level below which merging is profitable, denoted $s_{c r}$, first increases and then decreases in $\lambda$. This result is intimately linked to the price result discussed above. When $\lambda$ is small, an increase in $\lambda$ increases the price of the merging stores and this raises merger profitability. Eventually when $\lambda$ is relatively large, further increases in $\lambda$ make products too similar and this fosters competition. In this case, as $\lambda$ increases a smaller search cost is needed to make merging unprofitable. 


\subsection{Other symmetric equilibria}

All the discussion so far has been on a symmetric equilibrium with the merging firms charging a higher price than the non-merging firms and, correspondingly, consumers starting their search for satisfactory products at the non-merging stores. This equilibrium is the natural extension of the equilibrium that arises under perfect information (Deneckere and Davidson, 1985) and exists for all (reasonable) levels of the search cost. For this reason, it has been the focal point of the analysis so far.

However, as we mentioned above when we discussed the potential problems associated with the indeterminacy of consumer beliefs about which type of firms charge the lowest prices (cf. footnote 5), another symmetric equilibrium can be proposed. In such alternative symmetric equilibrium, consumers hold the belief that the merging stores charge prices lower than those of the non-merging firms and, correspondingly, they start their search for satisfactory products at the merging firms; firms respond by setting prices in such a way that consumer beliefs are fulfilled.

In this Section we focus attention on such an alternative equilibrium. Our first observation is that this alternative symmetric equilibrium is somewhat counterintuitive. The reason is that, due to the internalization-of-pricing externalities effect, we expect the merging firms to charge higher, rather than

lower, prices than the non-merging firms. Therefore, if such an equilibrium exists, it must be because the power of consumer beliefs at dictating firm pricing is sufficiently strong so as to more than offset the internalization-of-pricing externalities effect. Can this occur for all parameters? We do not expect it. From the received theory we know that when the search cost is exactly equal to zero (Deneckere and Davidson, 1985), such price ranking is impossible. By "continuity" we expect this alternative equilibrium to fail to exist when the search cost is positive but small. This is indeed what our next result shows. In addition, we can show that the same is true when the search cost is very high or for example when the number of firms is 3 .

Proposition 5. Assume that $k$ firms merge. Then a symmetric Nash equilibrium where $\hat{p}^{*}<\tilde{p}^{*}$ so that consumers start searching at the merged stores and then proceed by searching at the non-merging stores does not exist whenever one of the following conditions holds: (a) the search cost is sufficiently low, (b) the search cost is sufficiently high, (c) $n=3$, (d) the number of competitors is sufficiently large. As a result, in all these cases, the equilibrium in Proposition 1 is unique.

The proof of this result is in the Appendix. There we first derive the payoff functions of the merging and non-merging firms assuming that $\hat{p}^{*}<\tilde{p}^{*}$. Then we show that when search cost is either sufficiently high or sufficiently low, profit maximizing firms charge prices such that $\hat{p}^{*}>\tilde{p}^{*}$, which leads to a contradiction. This result implies that the alternative equilibrium where merging firms are visited first can only exist for intermediate levels of the search cost, which casts doubts about the appeal of the equilibrium. In fact, taking such an alternative equilibrium seriously requires consumer beliefs to be discontinuous in search costs, which is difficult to justify.

The intuition behind Propoition 5 is as follows. The price ranking of the firms is the outcome of the tension between the search-order effect, which pushes merging firms that are visited first to lower prices relative to the non-merging firms, and the internalization-of-pricing-externalities effect, which works in the opposite direction. The magnitude of the search cost affects the outcome of this tension. In fact, note 
that the search-order effect is practically non-existent when the search cost is arbitrarily close to zero, while the internalization-of-pricing-externalities effect is the strongest. In this case, the second effect has a dominating influence and this explains the result. When the search cost increases, the search-order effect gains importance, while the internalization-of-pricing-externalities effect loses strength. For intermediate levels of the search cost the equilibrium may exist (though not necessarily as demonstrated for the case $n=3)$. Finally, when search costs are very high, prices, whether form merging or not merging firms, are close to monopoly prices and the search-order effect loses again against the internalization-of-pricingexternalities effect.

We have explored alternative ways to affect the trade-off between the search-order effect and the internalization-of-pricing-externalities effect and rule out the alternative equilibrium where the merging firms charge lower prices and are visited first. What is important is to weaken the power consumer beliefs have at dictating equilibrium prices. For example, one can show that this equilibrium fails to exist when there is a sufficiently large number of consumers who have perfect information. The equilibrium in Proposition 1 by contrast survives this modification as well as our main result in Proposition 4 .

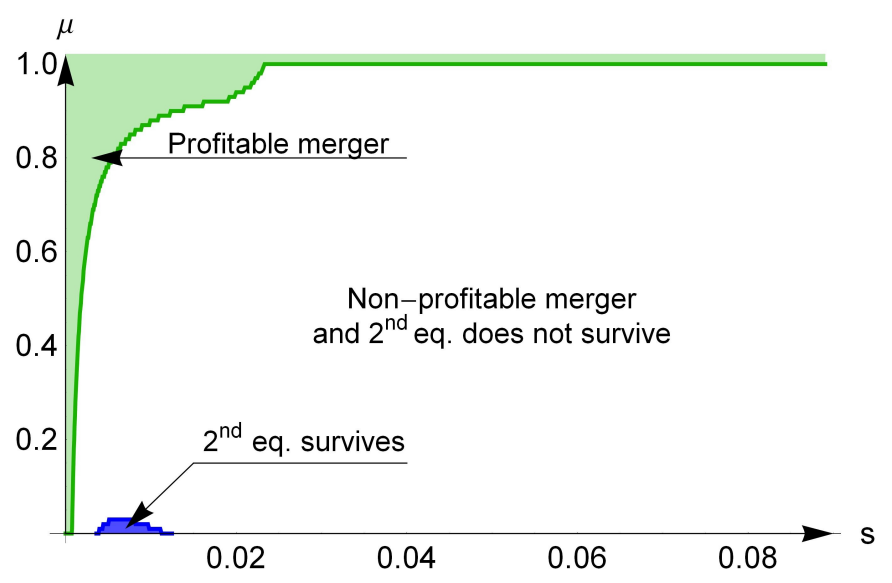

Figure 4: Model with a fraction $\mu$ of zero-search-cost consumers

To illustrate this last point, we provide next the outcome of the numerical analysis of a slightly modified model where we partition the set of consumers into two fractions: a fraction of the consumers $\mu$ have zero search costs while the rest of the consumers have positive search costs. The rest of the model remains exactly the same. In Figure 4 we set $n=7$ and plot the (blue) region of parameters for which an equilibrium where a 2 -firm merger charges a price lower than the price of the non-merging firms exists. The graph clearly shows the observation in Proposition 5 that, when all consumers have positive search costs, this equilibrium only exists for intermediate levels of the search cost. As we increase the fraction of consumers who have zero search cost, the equilibrium fails to exist. The equilibrium in Proposition 1 by contrast exists for all parameters and it can be seen that no matter the level of $\mu$, a merger is unprofitable provided that the search cost is sufficiently large. 


\section{Concluding remarks}

This paper has studied the role of search costs for merger profitability. We have used a model where firms compete in prices to sell differentiated products and consumers search sequentially to find price and product fit information. When the search cost is set equal to zero, the model gives the results in Deneckere and Davidson (1985). But when search costs are sizable, the price divergence between merging and nonmerging firms has implications for the path consumers follow when they search for satisfactory products. Since optimal consumer search prescribes the consumers to visit first the cheaper stores, the merging firms lose a lot of custom when search costs are relatively high. Our main result is that merging becomes unprofitable when the search cost is sufficiently large. The paper thus shows that a merger paradox can also arise when firms compete in prices to sell differentiated products. The paradox arises because a merger pushes the merging stores all the way back in the search order of consumers.

In the analysis of this paper we have followed the tradition and studied the implications of jointdecision making. By doing this, the paper has focussed on the short-run effects of mergers and has therefore abstracted from a number of issues that are relevant to mergers in the long-run. These issues include all type of business organizational changes aimed at delivering cost reductions. Arguably, in situations where search costs are important, these organizational changes may even include the shutting down of shops and the crowding of products together, which have the potential to generate beneficial search economies for the consumers. These long-run considerations are studied in a separate paper (Moraga-González and Petrikaitè, 2011). There we show that search cost economies may render a merger beneficial for consumers. 


\section{Appendix}

Proof of Proposition 1. The proof of the proposition is organized in three Claims. The first Claim shows that there is a pair of prices $\left\{\hat{p}^{*}, \tilde{p}^{*}\right\}$ that satisfies the system of first order conditions (12) and (13). The second claim shows that such a pair of prices is unique. Finally, the third Claim demonstrates that $\tilde{p}^{*}<\hat{p}^{*}$.

Claim 1. There is at least one pair of prices $\left\{\hat{p}^{*}, \tilde{p}^{*}\right\}$ that satisfies (12) and (13).

Proof. We first rewrite the FOC (12) as $G(\hat{p}, \tilde{p})=0$, where

$$
G(\hat{p}, \tilde{p}) \equiv \frac{1-\bar{x}^{k}}{k \bar{x}^{k-1}}-\hat{p}+g(\hat{p}, \tilde{p})
$$

and

$$
g(\hat{p}, \tilde{p}) \equiv \frac{\int_{0}^{\bar{x}-\hat{p}}(\varepsilon+\hat{p})^{k-2}(\varepsilon+\tilde{p})^{n-k}(\varepsilon+k \hat{p}) d \varepsilon}{(\bar{x}-\hat{p}+\tilde{p})^{n-k} \bar{x}^{k-1}}
$$

The FOC $G(\hat{p}, \tilde{p})=0$ defines an implicit relationship between $\hat{p}$ and $\tilde{p}$. Let the function $\eta_{1}(\tilde{p})$ define this relationship. This function is represented in Figure 5 below. By the implicit function theorem we have

$$
\frac{\partial \eta_{1}(\tilde{p})}{\partial \tilde{p}}=-\frac{\partial G / \partial \tilde{p}}{\partial G / \partial \hat{p}}=-\frac{\partial g / \partial \tilde{p}}{\partial g / \partial \hat{p}-1},
$$

The numerator of (15) is positive. This is because

$$
\frac{\partial g}{\partial \tilde{p}}=\frac{n-k}{\bar{x}^{k-1}(\bar{x}-\hat{p}+\tilde{p})^{n-k+1}} \int_{0}^{\bar{x}-\hat{p}}(\varepsilon+\hat{p})^{k-2}(\varepsilon+k \hat{p})(\varepsilon+\tilde{p})^{n-k-1}(\bar{x}-\hat{p}-\varepsilon) d \varepsilon>0
$$

The denominator of (15) is however negative. To see this, we note first that

$$
\begin{aligned}
\bar{x}^{k-1}(\bar{x}-\hat{p}+\tilde{p})^{n-k+1}\left[\frac{\partial g}{\partial \hat{p}}-1\right] & =(n-k) \int_{0}^{\bar{x}-\hat{p}}(\varepsilon+\hat{p})^{k-2}(\varepsilon+\tilde{p})^{n-k}(\varepsilon+k \hat{p}) d \varepsilon \\
& +(\bar{x}-\hat{p}+\tilde{p})(k-1) \int_{0}^{\bar{x}-\hat{p}}(\varepsilon+\hat{p})^{k-3}(\varepsilon+\tilde{p})^{n-k}(2 \varepsilon+k \hat{p}) d \varepsilon \\
& -(\bar{x}-\hat{p}+\tilde{p})^{n-k+1} \bar{x}^{k-2}[2 \bar{x}+(k-1) \hat{p}] .
\end{aligned}
$$

Assuming $k>2$, let us take the derivative of the RHS of (16) with respect to $\bar{x}$. We obtain:

$$
\begin{aligned}
& (n-k) \bar{x}^{k-2}(\bar{x}-\hat{p}+\tilde{p})^{n-k}[\bar{x}+(k-1) \hat{p}]+(k-1) \int_{0}^{\bar{x}-\hat{p}}(\varepsilon+\hat{p})^{k-3}(\varepsilon+\tilde{p})^{n-k}(2 \varepsilon+k \hat{p}) d \varepsilon \\
& +(k-1)(\bar{x}-\hat{p}+\tilde{p})^{n-k+1} \bar{x}^{k-3}[2 \bar{x}+(k-2) \hat{p}]-2(\bar{x}-\hat{p}+\tilde{p})^{n-k+1} \bar{x}^{k-2} \\
& -(n-k+1)(\bar{x}-\hat{p}+\tilde{p})^{n-k} \bar{x}^{k-2}[2 \bar{x}+(k-1) \hat{p}]+(\bar{x}-\hat{p}+\tilde{p})^{n-k+1}(k-2) \bar{x}^{k-3}[2 \bar{x}+(k-1) \hat{p}]
\end{aligned}
$$

which is equal to

$$
\begin{aligned}
& \bar{x}^{k-3}(\bar{x}-\hat{p}+\tilde{p})^{n-k}[(n-k) \bar{x}[\bar{x}+(k-1) \hat{p}]+(k-1)(\bar{x}-\hat{p}+\tilde{p})[2 \bar{x}+(k-2) \hat{p}]- \\
& 2 \bar{x}(\bar{x}-\hat{p}+\tilde{p})-(n-k+1) \bar{x}[2 \bar{x}+(k-1) \hat{p}]-(\bar{x}-\hat{p}+\tilde{p})(k-2)[2 \bar{x}+(k-1) \hat{p}]] \\
& +(k-1) \int_{0}^{\bar{x}-\hat{p}}(\varepsilon+\hat{p})^{k-3}(\varepsilon+\tilde{p})^{n-k}(2 \varepsilon+k \hat{p}) d \varepsilon
\end{aligned}
$$


and can be simplified further to

$$
-\bar{x}^{k-2}(\bar{x}-\hat{p}+\tilde{p})^{n-k}[\bar{x}(n-k+2)+(k-1) \hat{p}]+(k-1) \int_{0}^{\bar{x}-\hat{p}}(\varepsilon+\hat{p})^{k-3}(\varepsilon+\tilde{p})^{n-k}(2 \varepsilon+k \hat{p}) d \varepsilon
$$

If we now take the derivative of (18) with respect to $\bar{x}$ we obtain

$$
\begin{aligned}
& -\left[(k-2) \bar{x}^{k-3}(\bar{x}-\hat{p}+\tilde{p})^{n-k}+(n-k)(\bar{x}-\hat{p}+\tilde{p})^{n-k-1} \bar{x}^{k-2}\right][\bar{x}(n-k+2)+(k-1) \hat{p}] \\
& -(n-k+2) \bar{x}^{k-2}(\bar{x}-\hat{p}+\tilde{p})^{n-k}+(k-1) \bar{x}^{k-3}(\bar{x}-\hat{p}+\tilde{p})^{n-k}[2 \bar{x}+(k-2) \hat{p}]
\end{aligned}
$$

Putting terms together and simplifying, this is equal to

$$
-(n-k) \bar{x}^{k-2}(\bar{x}-\hat{p}+\tilde{p})^{n-k-1}[\bar{x}(n+1)+\tilde{p}(k-1)]<0 .
$$

This implies that the derivative of the RHS of (16) with respect to $\bar{x}$, given in equation (18), is decreasing in $\bar{x}$. Setting $\bar{x}$ equal to its lowest value, $\hat{p}$, in (18) gives

$$
-\hat{p}^{k-2} \tilde{p}^{n-k}[\hat{p}(n-k+2)+(k-1) \hat{p}]<0 .
$$

As a result, the RHS of (16) is also decreasing in $\bar{x}$. If we set now $\bar{x}=\hat{p}$ in the RHS of (16), we obtain $-\tilde{p}^{* n-k+1} \hat{p}^{k-1}(k+1)<0$. From this we conclude that $(16)$ is negative. As a result, since the numerator of $\partial \eta_{1}(\tilde{p}) / \partial \tilde{p}$ is positive and the denominator is negative, we infer that the function $\eta_{1}(\tilde{p})$ increases in $\tilde{p} .^{8}$

${ }^{8}$ When $k=2$, equation (16) changes slightly. Therefore, we treat this case separately. If $k=2$ then

and

$$
g(\hat{p}, \tilde{p})=\frac{\int_{0}^{\bar{x}-\hat{p}^{*}}(\varepsilon+\tilde{p})^{n-2}(\varepsilon+2 \hat{p}) d \varepsilon}{(\bar{x}-\hat{p}+\tilde{p})^{n-2} \bar{x}}
$$

$$
\frac{\partial g(\hat{p}, \tilde{p})}{\partial \hat{p}}=\frac{(n-2) \int_{0}^{\bar{x}-\hat{p}^{*}}(\varepsilon+\tilde{p})^{n-2}(\varepsilon+2 \hat{p}) d \varepsilon}{(\bar{x}-\hat{p}+\tilde{p})^{n-1} \bar{x}}+\frac{2 \int_{0}^{\bar{x}-\hat{p}^{*}}(\varepsilon+\tilde{p})^{n-2} d \varepsilon}{(\bar{x}-\hat{p}+\tilde{p})^{n-2} \bar{x}}-\frac{\bar{x}+\hat{p}}{\bar{x}}
$$

Then equation (16) is

$$
\begin{aligned}
\bar{x}(\bar{x}-\hat{p}+\tilde{p})^{n-1}\left[\frac{\partial g}{\partial \hat{p}}-1\right] & =(n-2) \int_{0}^{\bar{x}-\hat{p}}(\varepsilon+\tilde{p})^{n-2}(\varepsilon+2 \hat{p}) d \varepsilon \\
& +\frac{2}{n-1}(\bar{x}-\hat{p}+\tilde{p})^{n}-\frac{2}{n-1} \tilde{p}^{n-1}(\bar{x}-\hat{p}+\tilde{p})-(2 \bar{x}+\hat{p})(\bar{x}-\hat{p}+\tilde{p})^{n-1}
\end{aligned}
$$

The derivative of the RHS of (19) with respect to $\bar{x}$ is negative

$$
\begin{array}{r}
(n-2)(\bar{x}-\hat{p}+\tilde{p})^{n-2}(\bar{x}+\hat{p})+\frac{2 n}{n-1}(\bar{x}-\hat{p}+\tilde{p})^{n-1}-\frac{2}{n-1} \tilde{p}^{* n-1}- \\
2(\bar{x}-\hat{p}+\tilde{p})^{n-1}-(n-1)(2 \bar{x}+\hat{p})(\bar{x}-\hat{p}+\tilde{p})^{n-2}= \\
(\bar{x}-\hat{p}+\tilde{p})^{n-2}\left[(n-2) \bar{x}+(n-2) \hat{p}+\frac{2 n}{n-1} \bar{x}-\frac{2 n}{n-1} \hat{p}+\right. \\
\left.\frac{2 n}{n-1} \tilde{p}-2 \bar{x}+2 \hat{p}-2 \tilde{p}-2(n-1) \bar{x}-(n-1) \hat{p}\right]-\frac{2}{n-1} \tilde{p}^{n-1}= \\
-(\bar{x}-\hat{p}+\tilde{p})^{n-2}\left[\bar{x} \frac{n^{2}-n-2}{n-1}+\hat{p} \frac{n+1}{n-1}-\frac{2}{n-1} \tilde{p}\right]-\frac{2}{n-1} \tilde{p}^{n-1}< \\
-(\bar{x}-\hat{p}+\tilde{p})^{n-2}\left[\bar{x} \frac{n^{2}-n-2}{n-1}+\hat{p} \frac{n+1}{n-1}-\frac{2}{n-1} \bar{x}\right]-\frac{2}{n-1} \tilde{p}^{n-1}= \\
-(\bar{x}-\hat{p}+\tilde{p})^{n-2}\left[\bar{x} \frac{n^{2}-n-4}{n-1}+\hat{p} \frac{n+1}{n-1}\right]-\frac{2}{n-1} \tilde{p}^{n-1}<0
\end{array}
$$

Since this expression is negative, the same arguments can be used to conclude that $\partial G / \partial \tilde{p}$ is positive also when $k=2$, which implies that $\eta_{1}(\tilde{p})$ increases in $\tilde{p}$. 
Now consider the other equilibrium condition. Let us denote the LHS of (13) as $H(\hat{p}, \tilde{p})$. The condition $H(\hat{p}, \tilde{p})=0$ also defines an implicit relationship between $\hat{p}$ and $\tilde{p}$. Let the function $\eta_{2}(\tilde{p})$ define such relationship. This function is represented in Figure 5 below. By the implicit function theorem we have

$$
\frac{\partial \eta_{2}(\tilde{p})}{\partial \tilde{p}}=-\frac{\partial H / \partial \tilde{p}}{\partial H / \partial \hat{p}}
$$

We note that $H$ increases in $\hat{p}$. In fact,

$$
\begin{aligned}
\frac{\partial H}{\partial \hat{p}} & =(n-k)(\bar{x}-\hat{p}+\tilde{p})^{n-k-1}+(n-k) k \int_{0}^{\bar{x}-\hat{p}}(\varepsilon+\tilde{p})^{n-k-1}(\varepsilon+\hat{p})^{k-1} d \varepsilon \\
& -(n-k)(\bar{x}-\hat{p}+\tilde{p})^{n-k-1} \bar{x}^{k} \\
& =(n-k)(\bar{x}-\hat{p}+\tilde{p})^{n-k-1}\left(1-\bar{x}^{k}\right)+(n-k) k \int_{0}^{\bar{x}-\hat{p}}(\varepsilon+\tilde{p})^{n-k-1}(\varepsilon+\hat{p})^{k-1} d \varepsilon>0
\end{aligned}
$$

Moreover, $H$ decreases in $\tilde{p}$. In fact, for $k<n-1$ we have

$$
\begin{aligned}
\frac{\partial H}{\partial \tilde{p}} & =-\frac{1-\bar{x}^{n-k}}{1-\bar{x}}-(n-k)(\bar{x}-\hat{p}+\tilde{p})^{n-k-1}+(n-k)(n-k-1) \int_{0}^{\bar{x}-\hat{p}}(\varepsilon+\tilde{p})^{n-k-2}(\varepsilon+\hat{p})^{k} d \varepsilon \\
& <-\frac{1-\bar{x}^{n-k}}{1-\bar{x}}-(n-k)(\bar{x}-\hat{p}+\tilde{p})^{n-k-1}+(n-k) \bar{x}^{k}(n-k-1) \int_{0}^{\bar{x}-\hat{p}}(\varepsilon+\tilde{p})^{n-k-2} d \varepsilon \\
& =-\frac{1-\bar{x}^{n-k}}{1-\bar{x}}-(n-k)(\bar{x}-\hat{p}+\tilde{p})^{n-k-1}+(n-k) \bar{x}^{k}(\bar{x}-\hat{p}+\tilde{p})^{n-k-1}-(n-k) \bar{x}^{k} \tilde{p}^{n-k-1} \\
& =-\frac{1-\bar{x}^{n-k}}{1-\bar{x}}-(n-k)(\bar{x}-\hat{p}+\tilde{p})^{n-k-1}\left(1-\bar{x}^{k}\right)-(n-k) \bar{x}^{k} \tilde{p}^{n-k-1}<0,
\end{aligned}
$$

while for $k=n-1$ we get $\partial H / \partial \tilde{p}=-2<0$. As a result, we conclude that the function $\eta_{2}$ is increasing in $\tilde{p}$.

Therefore both $\eta_{1}$ and $\eta_{2}$ increase in $\tilde{p}$. To show that at least one pair of prices $\left\{\hat{p}^{*}, \tilde{p}^{*}\right\}$ exists that satisfies the system of FOCs (12) and (13), we need to show that the functions $\eta_{1}$ and $\eta_{2}$ cross at least once in the space $[0 ; 1 / 2] \times\left[0, p_{k}^{m}\right]$. As shown in Figure 5 we observe that $\eta_{1}(0)>0$. For this, note that

$$
G(\hat{p}, 0)=\frac{1-\bar{x}^{k}}{k \bar{x}^{k-1}}-\hat{p}+\frac{1}{\bar{x}^{k-1}(\bar{x}-\hat{p})^{n-k}} \int_{0}^{\bar{x}-\hat{p}}(\varepsilon+\hat{p})^{k-2}(\varepsilon+k \hat{p}) \varepsilon^{n-k} d \varepsilon .
$$

We have shown above that $G$ decreases in $\hat{p}$. Therefore, since

$$
G(0,0)=\frac{1-\bar{x}^{k}}{k \bar{x}^{k-1}}+\frac{1}{\bar{x}^{n-1}} \int_{0}^{\bar{x}} \varepsilon^{n-1} d \varepsilon>0,
$$

we conclude that $\eta_{1}(0)>0$.

On the contrary, we now observe that $\eta_{2}(0)<0$ (see Figure 5). This is because

$$
H(\hat{p}, 0)=1-(\bar{x}-\hat{p})^{n-k}+(n-k) \int_{0}^{\bar{x}-\hat{p}} \varepsilon^{n-k-1}(\varepsilon+\hat{p})^{k} d \varepsilon
$$

in increasing in $\hat{p}$ and $H(0,0)=1-\bar{x}^{n-k}+(n-k) \int_{0}^{\bar{x}} \varepsilon^{n-1} d \varepsilon>0$. 
Secondly, as depicted in Figure 5, we show that $\eta_{1}(1 / 2)<p_{k}^{m}<\eta_{2}(1 / 2)$, which ensures that the functions $\eta_{1}$ and $\eta_{2}$ cross at least once in the area $[0 ; 1 / 2] \times\left[0 ; p_{k}^{m}\right]$. To see that $\eta_{2}(1 / 2)>p_{k}^{m}$, we first calculate

$$
H\left(p_{k}^{m}, \frac{1}{2}\right)=1-\frac{1-\bar{x}^{n-k}}{1-\bar{x}} \frac{1}{2}-\left(\bar{x}-p_{k}^{m}+\frac{1}{2}\right)^{n-k}+(n-k) \int_{0}^{\bar{x}-p_{k}^{m}}\left(\varepsilon+\frac{1}{2}\right)^{n-k-1}\left(\varepsilon+p_{k}^{m}\right)^{k} d \varepsilon .
$$

Now we argue that $H\left(p_{k}^{m}, \frac{1}{2}\right)<0$. For this, we take the partial derivative of $H\left(p_{k}^{m}, \frac{1}{2}\right)$ with respect to $\bar{x}$ we get

$$
-\frac{1-(n-k) \bar{x}^{n-k-1}+(n-k-1) \bar{x}^{n-k}}{2(1-\bar{x})^{2}}-(n-k)\left(\bar{x}-p_{k}^{m}+\frac{1}{2}\right)^{n-k-1}\left(1-\bar{x}^{k}\right)<0,
$$

where the inequality follows from noting that the expression $1-(n-k) \bar{x}^{n-k-1}+(n-k-1) \bar{x}^{n-k}$ decreases in $\bar{x}$ and therefore it is higher than when we set $\bar{x}=1$, that is, $1-(n-k) \bar{x}^{n-k-1}+(n-k-1) \bar{x}^{n-k} \geq$ $1-(n-k)+(n-k-1)=0$. We then conclude that $H\left(p_{k}^{m}, \frac{1}{2}\right)$ is decreasing in $\bar{x}$.

Setting $\bar{x}$ equal to its lowest possible value we get

$$
\left.H\left(p_{k}^{m}, \frac{1}{2}\right)\right|_{\bar{x}=p_{k}^{m}}=1-\frac{1}{2^{n-k}}-\frac{1-\left(p_{k}^{m}\right)^{n-k}}{2\left(1-p_{k}^{m}\right)}
$$

This expression is decreasing in $n$. In fact, its derivative with respect to $n$ can be written as

$$
\begin{aligned}
& \frac{2^{n-k-1}\left(p_{k}^{m}\right)^{n-k} \ln p_{k}^{m}+\left(1-p_{k}^{m}\right) \ln 2}{2^{n-k}\left(1-p_{k}^{m}\right)} \\
& <\frac{1}{2^{n-k}\left(1-p_{k}^{m}\right)}\left[\left.p_{k}^{m} \ln p_{k}^{m}\left(2 p_{k}^{m}\right)^{n-k-1}\right|_{n=k+1}+\left(1-p_{k}^{m}\right) \ln 2\right] \\
& =\frac{1}{2^{n-k}\left(1-p_{k}^{m}\right)}\left[p_{k}^{m} \ln p_{k}^{m}+\left(1-p_{k}^{m}\right) \ln 2\right]<0
\end{aligned}
$$

The last inequatility follows from the fact that $p_{k}^{m} \ln p_{k}^{m}+\left(1-p_{k}^{m}\right) \ln 2<0$. This can be shown in three steps. We check the sign of the expression with the lowest and highest values of $k$ :

$$
\begin{gathered}
p_{k}^{m} \ln p_{k}^{m}+\left.\left(1-p_{k}^{m}\right) \ln 2\right|_{k=2}=\frac{1}{\sqrt{3}} \ln \frac{1}{\sqrt{3}}+\left(1-\frac{1}{\sqrt{3}}\right) \ln 2<0 \\
\lim _{k \rightarrow \infty}\left[p_{k}^{m} \ln p_{k}^{m}+\left(1-p_{k}^{m}\right) \ln 2\right]=0
\end{gathered}
$$

Let us now take the derivative of $p_{k}^{m} \ln p_{k}^{m}+\left(1-p_{k}^{m}\right) \ln 2$ with respect to $k$. We obtain the following: $\left(\partial p_{k}^{m} / \partial k\right)\left(\ln p_{k}^{m}+1-\ln 2\right)$. The $\operatorname{sign}$ of this expression depends on the $\operatorname{sign}$ of $1-\ln 2-\ln p_{k}^{m}=1-$ $\ln 2-\frac{1}{k} \ln (1+k)$, which is monotonically increasing in $k$, first negative and then positive. As a result, $p_{k}^{m} \ln p_{k}^{m}+\left(1-p_{k}^{m}\right) \ln 2$ first decreases and then increases in $k$. Together with the two facts above, we conclude it is always negative.

Since $\left.H\left(p_{k}^{m}, \frac{1}{2}\right)\right|_{\bar{x}=p_{k}^{m}}$ is decreasing in $n$, if we set $n$ equal to its lowest possible value, $k+1$, in $(21)$ we obtain

$$
\left.H\left(p_{k}^{m}, \frac{1}{2}\right)\right|_{\bar{x}=p_{k}^{m}} \leq\left. H\left(p_{k}^{m}, \frac{1}{2}\right)\right|_{\bar{x}=p_{k}^{m} ; n=k+1}=1-\frac{1}{2^{k+1-k}}-\frac{1-\left(p_{k}^{m}\right)^{k+1-k}}{2\left(1-p_{k}^{m}\right)}=1-\frac{1}{2}-\frac{1}{2}=0
$$


Therefore, since $H\left(p_{k}^{m}, \frac{1}{2}\right)$ is decreasing in $\bar{x}$, we conclude that $H\left(p_{k}^{m}, \frac{1}{2}\right)$ is always negative. And because $H$ is increasing in $\hat{p}$, we obtain the result that $\eta_{2}(1 / 2)>p_{k}^{m}$.

We now show that $\eta_{1}(1 / 2)<p_{k}^{m}$. Since $G$ is decreasing in $\hat{p}$, it suffices to demonstrate that

$$
G\left(p_{k}^{m}, \frac{1}{2}\right)=\frac{1-\bar{x}^{k}}{k \bar{x}^{k-1}}-p_{k}^{m}+\frac{\int_{0}^{\bar{x}-p_{k}^{m}}\left(\varepsilon+p_{k}^{m}\right)^{k-2}\left(\varepsilon+\frac{1}{2}\right)^{n-k}\left(\varepsilon+k p_{k}^{m}\right) d \varepsilon}{\left(\bar{x}-p_{k}^{m}+\frac{1}{2}\right)^{n-k} \bar{x}^{k-1}}<0
$$

Taking the derivative of $G\left(p_{k}^{m}, \frac{1}{2}\right)$ with respect to $n$ gives

$$
\begin{aligned}
& \left(\bar{x}-p_{k}^{m}+\frac{1}{2}\right)^{n-k} \bar{x}^{k-1} \frac{\partial G\left(p_{k}^{m}, \frac{1}{2}\right)}{\partial n} \\
& =\int_{0}^{\bar{x}-p_{k}^{m}}\left(\varepsilon+\frac{1}{2}\right)^{n-k}\left(\varepsilon+p_{k}^{m}\right)^{k-2}\left(\varepsilon+k p_{k}^{m}\right) \ln \left(\frac{\varepsilon+1 / 2}{\bar{x}-p_{k}^{m}+1 / 2}\right) d \varepsilon<0
\end{aligned}
$$

Since $G\left(p_{k}^{m}, \frac{1}{2}\right)$ decreases in $n$, we can set $n$ equal to its lowest value and write

$$
\begin{aligned}
G\left(p_{k}^{m}, \frac{1}{2}\right) & <\left.G\left(p_{k}^{m}, \frac{1}{2}\right)\right|_{n=k+1} \\
& =\frac{1-\bar{x}^{k}}{k \bar{x}^{k-1}}-p_{k}^{m}+\frac{1}{\bar{x}^{k-1}\left(\bar{x}-p_{k}^{m}+\frac{1}{2}\right)} \int_{0}^{\bar{x}-p_{k}^{m}}\left(\varepsilon+p_{k}^{m}\right)^{k-2}\left(\varepsilon+\frac{1}{2}\right)\left(\varepsilon+k p_{k}^{m}\right) d \varepsilon \\
& =\frac{1}{k \bar{x}^{k-1}\left(\bar{x}-p_{k}^{m}+\frac{1}{2}\right)} T(\bar{x})
\end{aligned}
$$

where

$$
T(\bar{x})=\left(\bar{x}-p_{k}^{m}+\frac{1}{2}\right)\left(1-\bar{x}^{k}-k \bar{x}^{k-1} p_{k}^{m}\right)+k \int_{0}^{\bar{x}-p_{k}^{m}}\left(\varepsilon+p_{k}^{m}\right)^{k-2}\left(\varepsilon+\frac{1}{2}\right)\left(\varepsilon+k p_{k}^{m}\right) d \varepsilon
$$
because

Note that $\frac{1}{k \bar{x}^{k-1}\left(\bar{x}-p_{k}^{m}+\frac{1}{2}\right)}>0$ Thus, $\left.G\left(p_{k}^{m}, \frac{1}{2}\right)\right|_{n=k+1}$ is negative if $T(\bar{x})<0 . T(\bar{x})$ decreases in $\bar{x}$

$$
\begin{aligned}
\frac{\partial T(\bar{x})}{\partial \bar{x}} & =1-\bar{x}^{k}-k \bar{x}^{k-1} p_{k}^{m}-\left(\bar{x}-p_{k}^{m}+\frac{1}{2}\right)\left(k \bar{x}^{k-1}+k(k-1) \bar{x}^{k-2} p_{k}^{m}\right) \\
& +k \bar{x}^{k-2}\left(\bar{x}-p_{k}^{m}+\frac{1}{2}\right)\left(\bar{x}+(k-1) p_{k}^{m}\right) \\
& =1-\bar{x}^{k}-k \bar{x}^{k-1} p_{k}^{m}
\end{aligned}
$$

and this expression decreases in $\bar{x}$. Therefore, using $\bar{x}=p_{k}^{m}$, we can write

$$
\frac{\partial T(\bar{x})}{\partial \bar{x}}<\left.\frac{\partial T(\bar{x})}{\partial \bar{x}}\right|_{\bar{x}=p_{k}^{m}}=1-\left(p_{k}^{m}\right)^{k}-k\left(p_{k}^{m}\right)^{k-1} p_{k}^{m}=0 .
$$

Since $T(\bar{x})$ decreases in $\bar{x}$ we then conclude that $T(\bar{x})<T\left(p_{k}^{m}\right)=0$. As a result the functions $\eta_{1}$ and $\eta_{2}$ cross at least once in the area $[0 ; 1 / 2] \times\left[0 ; p_{k}^{m}\right]$.

Claim 2. The pair of prices $\left\{\hat{p}^{*}, \tilde{p}^{*}\right\}$ that satisfies (12) and (13) is unique. 
Proof. To show this uniqueness result, it is enough to show that $\eta_{1}$ increases in $\tilde{p}$ at a rate less than 1 , while $\eta_{2}$ does so at a rate greater than 1 . From (15), since $\partial G / \partial \hat{p}<0$, we know that $\eta_{1}$ increases in $\tilde{p}$ if and only if $\partial G / \partial \hat{p}+\partial G / \partial \tilde{p}<0$. For the case $k>2$, we can then write

$$
\begin{aligned}
& \bar{x}^{k-1}(\bar{x}-\hat{p}+\tilde{p})^{n-k-1}\left[\frac{\partial G(\hat{p}, \tilde{p})}{\partial \tilde{p}}+\frac{\partial G(\hat{p}, \tilde{p})}{\partial \hat{p}}\right] \\
& =(n-k) \int_{0}^{\bar{x}-\hat{p}}(\varepsilon+\hat{p})^{k-2}(\varepsilon+k \hat{p})(\varepsilon+\tilde{p})^{n-k-1}(\bar{x}-\hat{p}-\varepsilon) d \varepsilon \\
& +(n-k) \int_{0}^{\bar{x}-\hat{p}}(\varepsilon+\hat{p})^{k-2}(\varepsilon+\tilde{p})^{n-k}(\varepsilon+k \hat{p}) d \varepsilon \\
& +(\bar{x}-\hat{p}+\tilde{p})(k-1) \int_{0}^{\bar{x}-\hat{p}}(\varepsilon+\hat{p})^{k-3}(\varepsilon+\tilde{p})^{n-k}(2 \varepsilon+k \hat{p}) d \varepsilon \\
& -(\bar{x}-\hat{p}+\tilde{p})^{n-k+1} \bar{x}^{k-2}[2 \bar{x}+(k-1) \hat{p}],
\end{aligned}
$$

which can be simplified to

$$
\begin{aligned}
& \bar{x}^{k-1}(\bar{x}-\hat{p}+\tilde{p})^{n-k}\left[\frac{\partial G(\hat{p}, \tilde{p})}{\partial \tilde{p}}+\frac{\partial G(\hat{p}, \tilde{p})}{\partial \hat{p}}\right] \\
& =(n-k) \int_{0}^{\bar{x}-\hat{p}}(\varepsilon+\hat{p})^{k-2}(\varepsilon+k \hat{p})(\varepsilon+\tilde{p})^{n-k-1} d \varepsilon \\
& +(k-1) \int_{0}^{\bar{x}-\hat{p}}(\varepsilon+\hat{p})^{k-3}(\varepsilon+\tilde{p})^{n-k}(2 \varepsilon+k \hat{p}) d \varepsilon-(\bar{x}-\hat{p}+\tilde{p})^{n-k} \bar{x}^{k-2}[2 \bar{x}+(k-1) \hat{p}] .
\end{aligned}
$$

We now notice that the RHS of (22) decreases in $\bar{x}$. In fact its derivative is

$$
\begin{aligned}
& (n-k) \bar{x}^{k-2}[\bar{x}+(k-1) \hat{p}](\bar{x}-\hat{p}+\tilde{p})^{n-k-1}+ \\
& +(k-1) \bar{x}^{k-3}(\bar{x}-\hat{p}+\tilde{p})^{n-k}[2 \bar{x}+(k-2) \hat{p}] \\
& -\left[(n-k)(\bar{x}-\hat{p}+\tilde{p})^{n-k-1} \bar{x}^{k-2}+(k-2)(\bar{x}-\hat{p}+\tilde{p})^{n-k} \bar{x}^{k-3}\right][2 \bar{x}+(k-1) \hat{p}] \\
& -2(\bar{x}-\hat{p}+\tilde{p})^{n-k} \bar{x}^{k-2}
\end{aligned}
$$

which, after rearranging, is equal to

$$
-(n-k) \bar{x}^{k-1}(\bar{x}-\hat{p}+\tilde{p})^{n-k-1}<0
$$


Therefore, if (22) is negative when setting $\bar{x}=\hat{p}$, then it is always negative, that is: ${ }^{9}$

$$
\bar{x}^{k-1}(\bar{x}-\hat{p}+\tilde{p})^{n-k}\left[\frac{\partial G(\hat{p}, \tilde{p})}{\partial \tilde{p}}+\frac{\partial G(\hat{p}, \tilde{p})}{\partial \hat{p}}\right]<-\tilde{p}^{n-k} \hat{p}^{k-1}(k+1)<0 .
$$

Similarly, using (20), since $\partial H / \partial \hat{p}>0$, we know that $\partial \eta_{2} / \partial \tilde{p}>1$ if and only if $\partial H / \partial \hat{p}+\partial H / \partial \tilde{p}<0$. For the case $k<n-1$, using the expressions above, we then compute

$$
\begin{aligned}
\frac{\partial H}{\partial \tilde{p}}+\frac{\partial H}{\partial \hat{p}} & =-\frac{1-\bar{x}^{n-k}}{1-\bar{x}}-(n-k)(\bar{x}-\hat{p}+\tilde{p})^{n-k-1}+(n-k)(n-k-1) \int_{0}^{\bar{x}-\hat{p}}(\varepsilon+\tilde{p})^{n-k-2}(\varepsilon+\hat{p})^{k} d \varepsilon \\
& +(n-k)(\bar{x}-\hat{p}+\tilde{p})^{n-k-1}\left(1-\bar{x}^{k}\right)+(n-k) k \int_{0}^{\bar{x}-\hat{p}}(\varepsilon+\tilde{p})^{n-k-1}(\varepsilon+\hat{p})^{k-1} d \varepsilon \\
& =-\frac{1-\bar{x}^{n-k}}{1-\bar{x}}+(n-k)(n-k-1) \int_{0}^{\bar{x}-\hat{p}}(\varepsilon+\tilde{p})^{n-k-2}(\varepsilon+\hat{p})^{k} d \varepsilon \\
& -(n-k)(\bar{x}-\hat{p}+\tilde{p})^{n-k-1} \bar{x}^{k}+(n-k) k \int_{0}^{\bar{x}-\hat{p}}(\varepsilon+\tilde{p})^{n-k-1}(\varepsilon+\hat{p})^{k-1} d \varepsilon
\end{aligned}
$$

This expression decreases in $\bar{x}$ because its partial derivative with respect to $\bar{x}$, after rearranging, is equal to

$$
-\frac{1-(n-k) \bar{x}^{n-k-1}+(n-k-1) \bar{x}^{n-k}}{(1-\bar{x})^{2}}
$$

and we have already shown above that the numerator of this expression is positive. Thus, using $\bar{x}=\hat{p}$ in (24) we can write ${ }^{10}$

$$
\frac{\partial H}{\partial \tilde{p}}+\frac{\partial H}{\partial \hat{p}}<-\frac{1-\hat{p}^{n-k}}{1-\hat{p}}-(n-k) \tilde{p}^{n-k-1} \hat{p}^{k}<0 .
$$

The result then follows.

Claim 3. The price charged by the merging stores is higher than the price of the non-merging ones, that is, $\hat{p}^{*}>\tilde{p}^{*}$.

${ }^{9}$ The same holds for the case when $k=2$. We have

$$
\begin{aligned}
\bar{x}(\bar{x}-\hat{p}+\tilde{p})^{n-2}\left[\frac{\partial G(\hat{p}, \tilde{p})}{\partial \tilde{p}}+\frac{\partial G(\hat{p}, \tilde{p})}{\partial \hat{p}}\right] & =(n-2) \int_{0}^{\bar{x}-\hat{p}}(\varepsilon+\tilde{p})^{n-3}(\varepsilon+2 \hat{p}) d \varepsilon \\
& +\frac{2}{n-1}(\bar{x}-\hat{p}+\tilde{p})^{n-1}-\frac{2}{n-1} \tilde{p}^{n-1}-(2 \bar{x}+\hat{p})(\bar{x}-\hat{p}+\tilde{p})^{n-2}
\end{aligned}
$$

The derivative of (23) with respect to $\bar{x}$ is negative because

$$
\begin{aligned}
& (n-2)(\bar{x}+\hat{p})(\bar{x}-\hat{p}+\tilde{p})^{n-3}+2(\bar{x}-\hat{p}+\tilde{p})^{n-2}-2(\bar{x}-\hat{p}+\tilde{p})^{n-2}-(n-2)(2 \bar{x}+\hat{p})(\bar{x}-\hat{p}+\tilde{p})^{n-3} \\
& =-\bar{x}(n-2)(\bar{x}-\hat{p}+\tilde{p})^{n-3}<0
\end{aligned}
$$

Then

$$
+\bar{x}(\bar{x}-\hat{p}+\tilde{p})^{n-2}\left[\frac{\partial G(\hat{p}, \tilde{p})}{\partial \tilde{p}}+\frac{\partial G(\hat{p}, \tilde{p})}{\partial \hat{p}}\right]<-3 \hat{p} \tilde{p}^{n-2}<0
$$

${ }^{10}$ If $k=n-1$ then

$$
\begin{aligned}
\frac{\partial H}{\partial \tilde{p}}+\frac{\partial H}{\partial \hat{p}} & =-2+\left(1-\bar{x}^{n-1}\right)+(n-1) \int_{0}^{\bar{x}-\hat{p}}(\varepsilon+\hat{p})^{n-2} d \varepsilon \\
& =-1+\bar{x}^{n-1}+\bar{x}^{n-1}-\hat{p}^{n-1}=-1-\hat{p}^{n-1}<0
\end{aligned}
$$


Proof. Let $\tilde{p}_{1}$ be the price at which the function $\eta_{1}$ crosses the 45 degrees line, i.e., $\eta_{1}\left(\tilde{p}_{1}\right)=\tilde{p}_{1}$; likewise, let $\tilde{p}_{2}$ be such that $\eta_{2}\left(\tilde{p}_{2}\right)=\tilde{p}_{2}\left(\tilde{p}_{1}\right.$ and $\tilde{p}_{2}$ are represented in Figure 5$)$. Given the properties of $\eta_{1}$ and $\eta_{2}$, if we show that $\tilde{p}_{1}>\tilde{p}_{2}$ then we can conclude that $\hat{p}^{*}>\tilde{p}^{*}$.

When $\tilde{p}=\tilde{p}_{1}$ from the FOC $G(\hat{p}, \tilde{p})=0$ we have:

$$
\tilde{p}_{1}=\frac{1-\bar{x}^{k}}{k \bar{x}^{k-1}}+\frac{\int_{0}^{\bar{x}-\tilde{p}_{1}}\left(\varepsilon+\tilde{p}_{1}\right)^{n-2}\left(\varepsilon+k \tilde{p}_{1}\right) d \varepsilon}{\bar{x}^{n-1}}
$$

Similarly, when $\tilde{p}=\tilde{p}_{2}$ the FOC $H(\hat{p}, \tilde{p})=0$ gives:

$$
1-\frac{1-\bar{x}^{n-k}}{1-\bar{x}} \tilde{p}_{2}-\bar{x}^{n-k}+(n-k) \int_{0}^{\bar{x}-\tilde{p}_{2}}\left(\varepsilon+\tilde{p}_{2}\right)^{n-1} d \varepsilon=0
$$

which can be rearranged as

$$
\tilde{p}_{2}=1-\bar{x}+\frac{1-\bar{x}}{1-\bar{x}^{n-k}}(n-k) \int_{0}^{\bar{x}-\tilde{p}_{2}}\left(\varepsilon+\tilde{p}_{2}\right)^{n-1} d \varepsilon
$$

For a contradiction, suppose that $\tilde{p}_{2}>\tilde{p}_{1}$. Then the difference between the RHS of (25) and the RHS of (26) must be negative. Let us denote this difference as $V$ and note that

$$
\begin{aligned}
V & \equiv \frac{1-\bar{x}^{k}}{k \bar{x}^{k-1}}+\frac{\int_{0}^{\bar{x}-\tilde{p}_{1}}\left(\varepsilon+\tilde{p}_{1}\right)^{n-2}\left(\varepsilon+k \tilde{p}_{1}\right) d \varepsilon}{\bar{x}^{n-1}}-1+\bar{x}-\frac{1-\bar{x}}{1-\bar{x}^{n-k}}(n-k) \int_{0}^{\bar{x}-\tilde{p}_{2}}\left(\varepsilon+\tilde{p}_{2}\right)^{n-1} d \varepsilon \\
& =\frac{\int_{0}^{\bar{x}-\tilde{p}_{1}}\left(\varepsilon+\tilde{p}_{1}\right)^{n-2}\left(\varepsilon+k \tilde{p}_{1}\right) d \varepsilon}{\bar{x}^{n-1}}+\frac{1+(k-1) \bar{x}^{k}-k \bar{x}^{k-1}}{k \bar{x}^{k-1}}-\frac{1-\bar{x}}{1-\bar{x}^{n-k}}(n-k) \int_{0}^{\bar{x}-\tilde{p}_{2}}\left(\varepsilon+\tilde{p}_{2}\right)^{n-1} d \varepsilon \\
& >\frac{\int_{0}^{\bar{x}-\tilde{p}_{1}}\left(\varepsilon+\tilde{p}_{1}\right)^{n-1} d \varepsilon}{\bar{x}^{n-1}}+\frac{1+(k-1) \bar{x}^{k}-k \bar{x}^{k-1}}{k \bar{x}^{k-1}}-\frac{1-\bar{x}}{1-\bar{x}^{n-k}}(n-k) \int_{0}^{\bar{x}-\tilde{p}_{2}}\left(\varepsilon+\tilde{p}_{2}\right)^{n-1} d \varepsilon
\end{aligned}
$$

where the inequality follows from replacing $\varepsilon+k \tilde{p}_{1}$ by $\varepsilon+\tilde{p}_{1}$ in the first integral.

Since the second integral in (27) is equal to $\left[\bar{x}^{n}-\left(\tilde{p}_{2}\right)^{n}\right] / n$, the whole expression in (27) increases in $\tilde{p}_{2}$. Therefore, $(27)$ must be higher than when we replace $\tilde{p}_{2}$ by $\tilde{p}_{1}$. That is, $(27)$ is higher than

$$
\begin{aligned}
& \frac{1}{\bar{x}^{n-1}} \int_{0}^{\bar{x}-\tilde{p}_{2}}\left(\varepsilon+\tilde{p}_{1}\right)^{n-1} d \varepsilon+\frac{1-(k-1) \bar{x}^{k}-k \bar{x}^{k-1}}{k \bar{x}^{k-1}}-\frac{1-\bar{x}}{1-\bar{x}^{n-k}}(n-k) \int_{0}^{\bar{x}-\tilde{p}_{2}}\left(\varepsilon+\tilde{p}_{1}\right)^{n-1} d \varepsilon \\
& =\frac{\bar{x}^{n}-\tilde{p}_{1}^{n}}{n}\left[\frac{1}{\bar{x}^{n-1}}-\frac{(1-\bar{x})(n-k)}{1-\bar{x}^{n-k}}\right]+\frac{1+(k-1) \bar{x}^{k}-k \bar{x}^{k-1}}{k \bar{x}^{k-1}}= \\
& =\frac{\bar{x}^{n}-\tilde{p}_{1}^{n}}{n}\left[\frac{1-\bar{x}^{n-k}-(n-k) \bar{x}^{n-1}+(n-k) \bar{x}^{n}}{\bar{x}^{n-1}\left(1-\bar{x}^{n-k}\right)}\right]+\frac{1+(k-1) \bar{x}^{k}-k \bar{x}^{k-1}}{k \bar{x}^{k-1}} \\
& =\frac{\bar{x}^{n}-\tilde{p}_{1}^{n}}{n\left(1-\bar{x}^{n-k}\right)}\left[\frac{1-\bar{x}^{n-k}-(n-k) \bar{x}^{n-1}(1-\bar{x})}{\bar{x}^{n-1}}\right]+\frac{1+(k-1) \bar{x}^{k}-k \bar{x}^{k-1}}{k \bar{x}^{k-1}}
\end{aligned}
$$

We now show that this last expression is positive, which establishes a contradiction. For this, we first note that the term in square brackets is positive. To see this, we take the derivative with respect to $\bar{x}$, which gives:

$$
n-k+(k-1) \bar{x}^{-k}-(n-1) \bar{x}^{-n}
$$

and note that this expression is decreasing in $n$ (its derivative is $\left.-\bar{x}^{-n}\left[1-\bar{x}^{n}-(n-1) \ln \bar{x}\right]<0\right)$. Then we can set $n=k+1$ and write that $n-k+(k-1) \bar{x}^{-k}-(n-1) \bar{x}^{-n}<1+(k-1) \bar{x}^{-k}-k \bar{x}^{-(k+1)}$. This 
last expression increases in $\bar{x}$ (its derivative is $k \bar{x}^{-(k+2)}[1+\bar{x}+k(1-\bar{x})]>0$ ) and therefore we can write $1+(k-1) \bar{x}^{-k}-k \bar{x}^{-(k+1)} \leq 0$. Therefore, the square bracket decreases in $\bar{x}$ so we can use the value $\bar{x}=1$ and show that

$$
\frac{1-\bar{x}^{n-k}-(n-k) \bar{x}^{n-1}(1-\bar{x})}{\bar{x}^{n-1}}>0
$$

Finally we observe that the last term of $(28)$ is also positive. This follows from the fact that the numerator $1+(k-1) \bar{x}^{k}-k \bar{x}^{k-1}$ decreases in $\bar{x}$ and then we can use the value $\bar{x}=1$ to write $1+(k-1) \bar{x}^{k}-k \bar{x}^{k-1}>0$.

Therefore we have proven that $V>0$, which is impossible if $\tilde{p}_{2}>\tilde{p}_{1}$. As a result, $\hat{p}^{*}>\tilde{p}^{*}$.

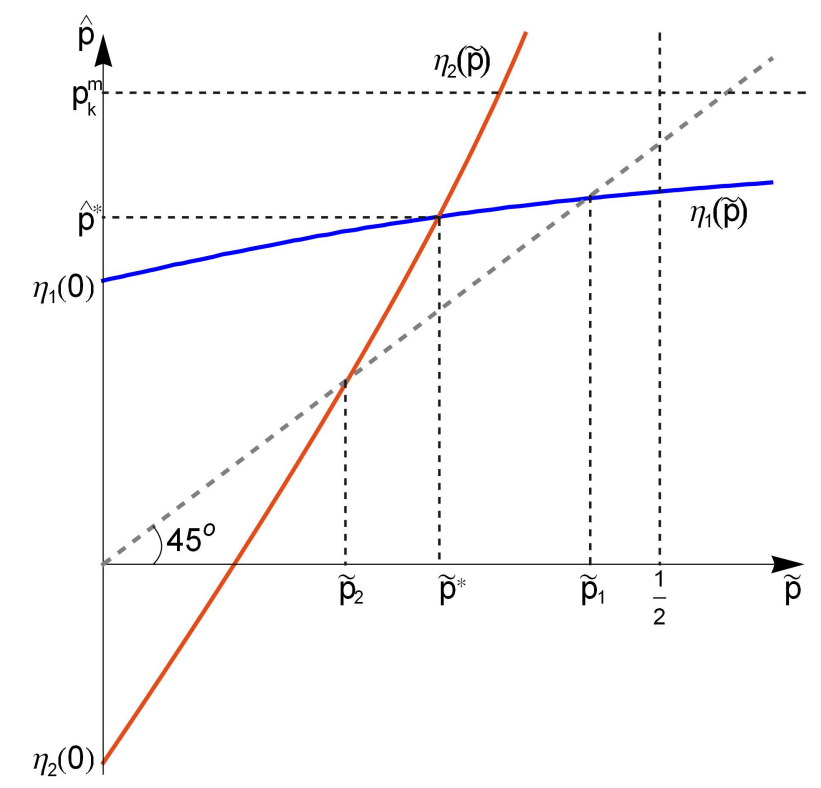

Figure 5: Existence and uniqueness of symmetric equilibrium

Proof of Proposition 2. Since we have already proven that $\hat{p}^{*}>\tilde{p}^{*}$, we focus on the inequality $\tilde{p}^{*}>p^{*}$. (a) Consider the case in which the search cost is sufficiently low, that is, the case when $\bar{x} \rightarrow 1$. We prove that $\tilde{p}^{*}>p^{*}$ by contradiction. Therefore, assume that, on the contrary, $\tilde{p}^{*}<p^{*}$ when $\bar{x} \rightarrow 1$. The aggregate quantity sold in the market by all firms together is $Q=1-\hat{p}^{* k} \tilde{p}^{* n-k}$. Using the FOCs and denoting the quantity sold by a non-merged firm by $\tilde{q}^{*}$, and that sold by all the merging firms together by $\hat{q}^{*}$ we can state that

$$
Y\left(\tilde{p}^{*}, \hat{p}^{*}\right) \equiv Q-(n-k) \tilde{q}^{*}-\hat{q}^{*}=0
$$

where

$$
\begin{gathered}
(n-k) \tilde{q}^{*}=\frac{1-\bar{x}^{n-k}}{1-\bar{x}} \tilde{p}^{*} \\
\hat{q}^{*}=k \hat{p}^{*} \bar{x}^{k-1}\left(\bar{x}-\hat{p}^{*}+\tilde{p}^{*}\right)^{n-k}-k(k-1) \hat{p}^{*} \int_{0}^{\bar{x}-\hat{p}^{*}}\left(\varepsilon+\hat{p}^{*}\right)^{k-2}\left(\varepsilon+\tilde{p}^{*}\right)^{n-k} d \varepsilon .
\end{gathered}
$$


We now argue that $Y\left(\tilde{p}^{*}, \hat{p}^{*}\right)$ is decreasing in $\tilde{p}^{*}$. This is because $\partial Q / \partial \tilde{p}^{*}<0, \partial \tilde{q}^{*} / \partial \tilde{p}^{*}>0$ and

$$
\begin{aligned}
\frac{1}{k(n-k) \hat{p}^{*}} \frac{\partial \hat{q}^{*}}{\partial \tilde{p}^{*}} & =\bar{x}^{k-1}\left(\bar{x}-\hat{p}^{*}+\tilde{p}^{*}\right)^{n-k-1}-(k-1) \int_{0}^{\bar{x}-\hat{p}^{*}}\left(\varepsilon+\hat{p}^{*}\right)^{k-2}\left(\varepsilon+\tilde{p}^{*}\right)^{n-k-1} d \varepsilon \\
& >\bar{x}^{k-1}\left(\bar{x}-\hat{p}^{*}+\tilde{p}^{*}\right)^{n-k-1}-\left(\bar{x}-\hat{p}^{*}+\tilde{p}^{*}\right)^{n-k-1}(k-1) \int_{0}^{\bar{x}-\hat{p}^{*}}\left(\varepsilon+\hat{p}^{*}\right)^{k-2} d \varepsilon \\
& =\left(\bar{x}-\hat{p}^{*}+\tilde{p}^{*}\right)^{n-k-1} \hat{p}^{* k-1}>0
\end{aligned}
$$

Next, since $Y$ is decreasing in $\tilde{p}^{*}$ and by assumption $\tilde{p}^{*}<p^{*}$ we must have $Y\left(p^{*}, \hat{p}^{*}\right)<0$. When $\bar{x} \rightarrow 1, Y\left(p^{*}, \hat{p}^{*}\right)$ goes to:

$$
1-\hat{p}^{* k} p^{* n-k}-(n-k) p^{*}-k \hat{p}^{*}\left(1-\hat{p}^{*}+p^{*}\right)^{n-k}+k(k-1) \hat{p}^{*} \int_{0}^{1-\hat{p}^{*}}\left(\varepsilon+\hat{p}^{*}\right)^{k-2}\left(\varepsilon+p^{*}\right)^{n-k} d \varepsilon
$$

Now we invoke the FOC of the merged entity, denoted above by $G\left(\hat{p}^{*}, \tilde{p}^{*}\right)$. The function $G\left(\hat{p}^{*}, \tilde{p}^{*}\right)$ was shown to be increasing in $\tilde{p}^{*}$ so when $\tilde{p}^{*}<p^{*}$ we must have $G\left(\hat{p}^{*}, p^{*}\right)>G\left(\hat{p}^{*}, \tilde{p}^{*}\right)=0$. Therefore:

$$
\begin{aligned}
\lim _{\bar{x} \rightarrow 1} G\left(\hat{p}^{*}, p^{*}\right) & \equiv-\hat{p}^{*}+\frac{1}{(1-\hat{p}+\tilde{p})^{n-k}} \int_{0}^{1-\hat{p}}(\varepsilon+\hat{p})^{k-2}(\varepsilon+\tilde{p})^{n-k}(\varepsilon+k \hat{p}) d \varepsilon \\
& =-\hat{p}^{*}+\frac{(k-1)}{\left(1-\hat{p}^{*}+\tilde{p}^{*}\right)^{n-k}} \hat{p}^{*} \int_{0}^{1-\hat{p}^{*}}\left(\varepsilon+\hat{p}^{*}\right)^{k-2}\left(\varepsilon+p^{*}\right)^{n-k} d \varepsilon \\
& +\frac{1}{\left(1-\hat{p}^{*}+\tilde{p}^{*}\right)^{n-k}} \int_{0}^{1-\hat{p}^{*}}\left(\varepsilon+\hat{p}^{*}\right)^{k-1}\left(\varepsilon+p^{*}\right)^{n-k} d \varepsilon
\end{aligned}
$$

must be positive, which implies that it must be the case that

$$
-k \hat{p}^{*}\left(1-\hat{p}^{*}+p^{*}\right)^{n-k}+k(k-1) \hat{p}^{*} \int_{0}^{1-\hat{p}^{*}}\left(\varepsilon+\hat{p}^{*}\right)^{k-2}\left(\varepsilon+p^{*}\right)^{n-k} d \varepsilon>-k \int_{0}^{1-\hat{p}^{*}}\left(\varepsilon+\hat{p}^{*}\right)^{k-1}\left(\varepsilon+p^{*}\right)^{n-k} d \varepsilon .
$$

Using this inequality in (29), we get that

$$
\lim _{\bar{x} \rightarrow 1} Y\left(p^{*}, \hat{p}^{*}\right)>1-\hat{p}^{* k} p^{* n-k}-(n-k) p^{*}-k \int_{0}^{1-\hat{p}^{*}}\left(\varepsilon+\hat{p}^{*}\right)^{k-1}\left(\varepsilon+p^{*}\right)^{n-k} d \varepsilon
$$

This last expression is increasing in $\hat{p}^{*}$. This is because the sign of its derivative with respect to $\hat{p}^{*}$ is the same as the sign of the following expression

$$
\begin{aligned}
& -\hat{p}^{* k-1} p^{* n-k}-(k-1) \int_{0}^{1-\hat{p}^{*}}\left(\varepsilon+\hat{p}^{*}\right)^{k-2}\left(\varepsilon+p^{*}\right)^{n-k} d \varepsilon+\left(1-\hat{p}^{*}+p^{*}\right)^{n-k} \\
& >-\hat{p}^{* k-1} p^{* n-k}-(k-1)\left(1-\hat{p}^{*}+p^{*}\right)^{n-k} \int_{0}^{1-\hat{p}^{*}}\left(\varepsilon+\hat{p}^{*}\right)^{k-2} d \varepsilon+\left(1-\hat{p}^{*}+p^{*}\right)^{n-k} \\
& =-\hat{p}^{* k-1} p^{* n-k}+\left(1-\hat{p}^{*}+p^{*}\right)^{n-k} \hat{p}^{* k-1}>0
\end{aligned}
$$

Therefore, (30) is greater than after setting $\hat{p}^{*}=p^{*}$, that is

$$
\begin{aligned}
\lim _{\bar{x} \rightarrow 1} Y\left(p^{*}, \hat{p}^{*}\right) & >1-\left(p^{*}\right)^{n}-(n-k) p^{*}-k\left[\frac{1}{n}-\frac{1}{n}\left(p^{*}\right)^{n}\right] \\
& =n p^{*}-(n-k) p^{*}-k p^{*}=0
\end{aligned}
$$


where for the last equality we have used the FOC of a firm in the pre-merger market. (If $\bar{x} \rightarrow 1$ then the first order condition of a firm in a pre-merger market becomes $1-n p^{*}-\left(p^{*}\right)^{n}=0$.) Consequently, if $\tilde{p}>p^{*}$ then we have $\lim _{\bar{x} \rightarrow 1} Y\left(p^{*}, \hat{p}\right)>0$, which establishes a contradiction.

(b) Consider now the case in which the search cost is sufficiently high, that is, $\bar{x} \rightarrow p_{k}^{m}$. From proposition 1 we know that the solution to the FOCs is unique. Therefore, if we find two prices for which (12) and (13) hold when $\bar{x} \rightarrow p_{k}^{m}$, then these prices are indeed the equilibrium prices. Let us take the limit of the LHS of (12) and (13) when $\bar{x} \rightarrow p_{k}^{m}$ and let us use the notation $\lim _{\bar{x} \rightarrow p_{k}^{m}} \tilde{p}^{*}=\tilde{p}_{l}$ and $\lim _{\bar{x} \rightarrow p_{k}^{m}} \hat{p}^{*}=p_{k}^{m}$. Then we get the following expressions

$$
\begin{aligned}
\left(\tilde{p}_{l}\right)^{n-k}\left[1-(k+1)\left(p_{k}^{m}\right)^{k}\right] & =0 \\
\left(1-p_{k}^{m}\right)\left(1-\tilde{p}_{l}^{n-k}\right)-\tilde{p}_{l}\left[1-\left(p_{k}^{m}\right)^{n-k}\right] & =0 .
\end{aligned}
$$

The first equation is indeed zero given the definition of $p_{k}^{m}$ and the second equation therefore gives the value of $\tilde{p}_{l}$ when $\bar{x} \rightarrow p_{k}^{m}$. We note that the price $\tilde{p}_{l}$ is less than $p^{m}=1 / 2$ because, as shown in the proof of proposition $1, H\left(p_{k}^{m}, 1 / 2\right) \leq 0$.

We are interested in comparing $\tilde{p}_{l}$ with the pre-merger equilibrium price. Let us use the notation $p_{l}=\lim _{\bar{x} \rightarrow p_{k}^{m}} p^{*}$. We now argue that $\tilde{p}_{l}>p_{l}$. To show this, we take the limit when $\bar{x} \rightarrow p_{k}^{m}$ of the FOC that determines $p_{l}$. This gives:

$$
\left(1-p_{k}^{m}\right)\left(1-p_{l}^{n}\right)-p_{l}\left[1-\left(p_{k}^{m}\right)^{n}\right]=0
$$

If we fix the value of $p_{k}^{m}$ then the solution of this equation, $p_{l}$, decreases with $n$. Comparing this equation with (31), since $n-k<n$, it is immediately clear that $\tilde{p}_{l}>p_{l}$.

(c) Finally, we look at the case $n=3$. If $n=3$ then the FOC of a merging firm

$$
\left(\bar{x}-\hat{p}^{*}+\tilde{p}^{*}\right)\left(1-\bar{x}^{2}-2 \hat{p}^{*} \bar{x}\right)+2 \int_{0}^{\bar{x}-\hat{p}^{*}}\left(\varepsilon+\tilde{p}^{*}\right)\left(\varepsilon+2 \hat{p}^{*}\right) d \varepsilon=0
$$

may be rearranged as follows

$$
\hat{p}^{* 3}-\hat{p}^{*} \bar{x}^{2}-\tilde{p}^{*}\left(3 \hat{p}^{* 2}-1\right)=\frac{\bar{x}^{3}}{3}-\frac{\hat{p}^{* 3}}{3}-\bar{x}+\hat{p}^{*} .
$$

The FOC of a non-merging firm

$$
1-\tilde{p}^{*}-\bar{x}+\hat{p}^{*}-\tilde{p}^{*}+\int_{0}^{\bar{x}-\hat{p}^{*}}\left(\varepsilon+\hat{p}^{*}\right)^{2} d \varepsilon=0
$$

gives us the relation

$$
\frac{\bar{x}^{3}}{3}-\frac{\hat{p}^{* 3}}{3}-\bar{x}+\hat{p}^{*}=2 \tilde{p}^{*}-1 .
$$

Using this in (32) we have

$$
\hat{p}^{* 3}-\hat{p}^{*} \bar{x}^{2}-3 \tilde{p}^{*} \hat{p}^{* 2}-\tilde{p}^{*}+1=0,
$$

or

$$
\tilde{p}^{*}=\frac{1+\hat{p}^{* 3}-\hat{p}^{*} \bar{x}^{2}}{1+3 \hat{p}^{* 2}} .
$$


From the FOC in the pre-merger market we get that

$$
p^{*}=\frac{1-p^{* 3}}{1+\bar{x}+\bar{x}^{2}} .
$$

Since, by strategic complementarity, $\tilde{p}^{*}$ increases in $\hat{p}^{*}$ and since $\hat{p}^{*}>p^{*}$, the difference $\tilde{p}^{*}-p^{*}$ is greater than when we replace $\hat{p}^{*}$ by $p^{*}$. Therefore

$$
\tilde{p}^{*}-p^{*}=\frac{1+\hat{p}^{* 3}-\hat{p}^{*} \bar{x}^{2}}{1+3 \hat{p}^{* 2}}-\frac{1-p^{* 3}}{1+\bar{x}+\bar{x}^{2}}>\frac{1+p^{* 3}-p^{*} \bar{x}^{2}}{1+3 p^{* 2}}-\frac{1-p^{* 3}}{1+\bar{x}+\bar{x}^{2}}
$$

The RHS of this expression is concave in $\bar{x}$ because its second derivative with respect to $\bar{x}$ is negative:

$$
-\frac{2 p^{*}}{1+3 p^{* 2}}-\frac{6\left(1-p^{* 3}\right) \bar{x}(1+\bar{x})}{\left(1+\bar{x}+\bar{x}^{2}\right)^{3}}<0
$$

Hence, if the RHS of (33) is positive with the highest and the lowest possible values of $\bar{x}$ then it is positive for all possible $\bar{x}$ values. Setting $\bar{x}=1$ in the RHS of (33) gives

$$
\frac{2-3 p^{*}-3 p^{* 2}+4 p^{* 3}+3 p^{* 5}}{3\left(1+3 p^{* 2}\right)} .
$$

which is always positive as shown in Figure 6.

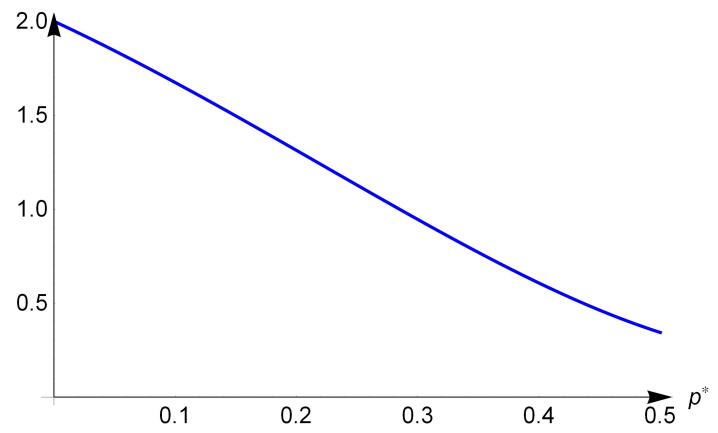

Figure 6: Plot of expression 34

Setting $\bar{x}=p^{*}$ in (33) gives

$$
\frac{p\left(1-3 p^{*}+3 p^{* 2}\right)}{1+3 p^{* 2}}>0
$$

Thus, $\tilde{p}^{*}>p^{*}$.

Proof of Proposition 3. We first prove that the function $\eta_{1}(\tilde{p})$ shifts downwards if $\bar{x}$ increases. From the relation $G\left(\eta_{1}(\tilde{p}), \tilde{p}, \bar{x}\right)=0$, we obtain that $\partial \eta_{1} / \partial \bar{x}=-(\partial G / \partial \bar{x}) /(\partial G / \partial \hat{p})$. Above in the proof of Proposition 1, we have already shown that $\partial G / \partial \hat{p}<0$. Therefore, we need to show that $\partial G / \partial \bar{x}<0$ where

$$
\begin{aligned}
\frac{\partial G}{\partial \bar{x}} & =-\frac{(k-1)\left(1-\bar{x}^{k}-k \hat{p} \bar{x}^{k-1}\right)}{k \bar{x}^{k}} \\
& -\frac{(k-1)(\bar{x}-\hat{p}+\tilde{p})+(n-k) \bar{x}}{\bar{x}^{k}(\bar{x}-\hat{p}+\tilde{p})^{n-k+1}} \int_{0}^{\bar{x}-\hat{p}}(\varepsilon+\hat{p})^{k-2}(\varepsilon+\tilde{p})^{n-k}(\varepsilon+k \hat{p}) d \varepsilon .
\end{aligned}
$$


Using the FOC $G(\hat{p}, \tilde{p})=0$ we can rewrite $\partial G / \partial \bar{x}$ as follows:

$$
\begin{aligned}
\frac{\partial G}{\partial \bar{x}} & =-\frac{(k-1)\left(1-\bar{x}^{k}-k \hat{p} \bar{x}^{k-1}\right)}{k \bar{x}^{k}}+\left[\frac{(k-1)(\bar{x}-\hat{p}+\tilde{p})+(n-k) \bar{x}}{\bar{x}^{k}(\bar{x}-\hat{p}+\tilde{p})^{n-k+1}}\right] \frac{(\bar{x}-\hat{p}+\tilde{p})^{n-k}\left(1-\bar{x}^{k}-k \bar{x}^{k-1} \hat{p}\right)}{k} \\
& =-\frac{\left(1-\bar{x}^{k}-k \hat{p} \bar{x}^{k-1}\right)}{k \bar{x}^{k}}\left[k-1-\frac{(k-1)(\bar{x}-\hat{p}+\tilde{p})+(n-k) \bar{x}}{(\bar{x}-\hat{p}+\tilde{p})}\right] \\
& =\frac{\left(1-\bar{x}^{k}-k \hat{p} \bar{x}^{k-1}\right)(n-k)}{k \bar{x}^{k-1}(\bar{x}-\hat{p}+\tilde{p})}<0
\end{aligned}
$$

where the last inequality follows form the fact that for $G(\hat{p}, \tilde{p})=0$ it must be the case that $1-\bar{x}^{k}-k \bar{x}^{k-1} \hat{p}<$ 0 . As a result, we conclude that $\eta_{1}(\tilde{p})$ shifts downwards when $\bar{x}$ increases.

We now show that $\eta_{2}(\tilde{p})$ shifts upwards when $\bar{x}$ increases. From the relation $H\left(\eta_{1}(\tilde{p}), \tilde{p}, \bar{x}\right)=0$, we obtain that $\partial \eta_{2} / \partial \bar{x}=-(\partial H / \partial \bar{x}) /(\partial H / \partial \hat{p})$. Above in the proof of Proposition 1, we have already shown that $\partial H / \partial \hat{p}>0$. Therefore, we need to show that $\partial H / \partial \bar{x}<0$. In fact, taking the derivative of $H$ with respect to $\bar{x}$ gives

$$
\frac{1}{(n-k)} \frac{\partial H}{\partial \bar{x}}=-\frac{\tilde{p}\left[1-(n-k) \bar{x}^{n-k-1}+(n-k-1) \bar{x}^{n-k}\right]}{(n-k)(1-\bar{x})^{2}}-(\bar{x}-\hat{p}+\tilde{p})^{n-k-1}\left(1-\bar{x}^{k}\right)<0,
$$

where the last inequality uses the fact that $1-(n-k) \bar{x}^{n-k-1}+(n-k-1) \bar{x}^{n-k}$ is decreasing in $\bar{x}$ and equals 0 when $\bar{x}=1$.

Since $\eta_{1}(\tilde{p})$ shifts downwards while $\eta_{2}(\tilde{p})$ shifts upwards when $\bar{x}$ increases, we conclude that $\hat{p}^{*}$ and $\tilde{p}^{*}$ decrease in $\bar{x}$ (or increase in $s$ ).

Proof of Proposition 4. Post-merger, the profit of a merging firm is $\hat{\pi}^{*} / k$, while pre-merger it is $\pi^{*}$. Then, we need to consider the difference $\hat{\pi}^{*} / k-\pi^{*}$.

(a) To prove this we set $k=2$ in the profits difference $\hat{\pi}^{*} / k-\pi^{*}$ and study its sign when $\bar{x} \rightarrow p_{k}^{m}(=$ $1 / \sqrt{3})$. Then, for $k=2$, the profit of a merging firm is

$$
\lim _{\bar{x} \rightarrow 1 / \sqrt{3}} \frac{\hat{\pi}^{*}}{2}=\frac{\left(\tilde{p}_{l}\right)^{n-2}}{3 \sqrt{3}}
$$

where we use the same notation as above in the proof of Proposition 2: $\tilde{p}_{l}=\lim _{\bar{x} \rightarrow p_{k}^{m}} \tilde{p}^{*}$. We have shown above that $\tilde{p}_{l}<p^{m}=1 / 2$. Therefore,

$$
\lim _{\bar{x} \rightarrow 1 / \sqrt{3}} \frac{\hat{\pi}^{*}}{2}<\frac{\left(p^{m}\right)^{n-2}}{3 \sqrt{3}}
$$

which implies that

$$
\lim _{\bar{x} \rightarrow 1 / \sqrt{3}}\left[\frac{\hat{\pi}^{*}}{2}-\pi^{*}\right]<\frac{(1 / 2)^{n-2}}{3 \sqrt{3}}-\frac{\left(p_{l}\right)^{2}\left[1-\left(p_{2}^{m}\right)^{n}\right]}{n\left(1-p_{2}^{m}\right)}
$$

where, again as in the proof of Proposition $2, p_{l}=\lim _{\bar{x} \rightarrow p_{k}^{m}} p^{*}$. If we demonstrate that

$$
\frac{\left(p_{l}\right)^{2}\left[1-\left(p_{2}^{m}\right)^{n}\right]}{n\left(1-p_{2}^{m}\right)}>\frac{(1 / 2)^{n-2}}{3 \sqrt{3}}
$$


or

$$
p_{l}>\sqrt{\frac{n\left(1-p_{2}^{m}\right)(1 / 2)^{n-2}}{\left[1-\left(p_{2}^{m}\right)^{n}\right] 3 \sqrt{3}}}=\sqrt{\frac{n\left(1-3^{-1 / 2}\right)(1 / 2)^{n-2}}{3 \sqrt{3}\left(1-3^{-n / 2}\right)}}
$$

then the result follows.

To show that (35) indeed holds, we observe that the pre-merger market FOC

$$
1-p^{* n}-p^{*} \frac{\left(1-\bar{x}^{n}\right)}{1-\bar{x}}=0
$$

which determines the value of $p^{*}$, is decreasing in $p^{*}$. Taking the limit of (36) when $\bar{x} \rightarrow 1 / \sqrt{3}$ gives

$$
1-\left(p_{l}\right)^{n}-p_{l} \frac{\left(1-3^{-n / 2}\right)}{1-3^{-1 / 2}}=0
$$

Now, using (35), if we replace $p_{l}$ by $\left[\frac{n\left(1-3^{-1 / 2}\right)}{2^{n-2} 3 \sqrt{3}\left(1-3^{-n / 2}\right)}\right]^{\frac{1}{2}}$ in this expression we get

$$
1-\left[\frac{n\left(1-3^{-1 / 2}\right)}{2^{n-2} 3 \sqrt{3}\left(1-3^{-n / 2}\right)}\right]^{\frac{n}{2}}-\sqrt{\frac{n\left(1-3^{-1 / 2}\right)}{2^{n-2} 3 \sqrt{3}\left(1-3^{-n / 2}\right)}} \frac{\left(1-3^{-n / 2}\right)}{1-3^{-1 / 2}}
$$

which is always positive as shown in Figure 7.

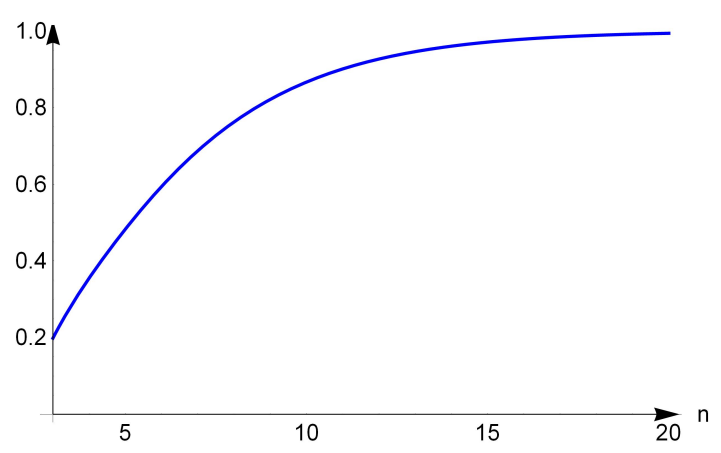

Figure 7: Plot of expression (37).

Since (36) is decreasing in $p^{*}$, then (35) must hold.

(b) Using the definition of $p_{k}^{m}$, we have that $1-\left(p_{k}^{m}\right)^{k}=k\left(p_{k}^{m}\right)^{k}$. Therefore we can write

$$
\begin{aligned}
\lim _{\bar{x} \rightarrow p_{k}^{m} ; n \rightarrow \infty}\left[\frac{\hat{\pi}^{*}}{k}-\pi^{*}\right] & <\lim _{n \rightarrow \infty}\left[\frac{p_{k}^{m}}{2^{n-k}}\left(1-\left(p_{k}^{m}\right)^{k}\right)-\frac{\left(p_{l}\right)^{2}\left(1-\left(p_{k}^{m}\right)^{n}\right)}{n\left(1-p_{k}^{m}\right)}\right] \\
& =\lim _{n \rightarrow \infty}\left[\frac{\left(p_{k}^{m}\right)^{k+1}}{2^{n-k}}-\frac{\left(p_{l}\right)^{2}\left(1-\left(p_{k}^{m}\right)^{n}\right)}{n\left(1-p_{k}^{m}\right)}\right]
\end{aligned}
$$

Note that $1-\left(p_{k}^{m}\right)^{n}>1-\left(p_{k}^{m}\right)^{k}=k\left(p_{k}^{m}\right)^{k}$. Thus,

$$
\begin{aligned}
& \lim _{n \rightarrow \infty}\left[\frac{\left(p_{k}^{m}\right)^{k+1}}{2^{n-k}}-\frac{\left(p_{l}\right)^{2}\left(1-\left(p_{k}^{m}\right)^{n}\right)}{n\left(1-p_{k}^{m}\right)}\right]<\lim _{n \rightarrow \infty}\left[\frac{\left(p_{k}^{m}\right)^{k+1}}{2^{n-k}}-\frac{\left(p_{l}\right)^{2} k\left(p_{k}^{m}\right)^{k}}{n\left(1-p_{k}^{m}\right)}\right]= \\
& \frac{\left(p_{k}^{m}\right)^{k}}{1-p_{k}^{m}} \lim _{n \rightarrow \infty}\left[\frac{p_{k}^{m}\left(1-p_{k}^{m}\right)}{2^{n-k}}-\frac{\left(p_{l}\right)^{2} k}{n}\right]=0,
\end{aligned}
$$


which shows that for any $k$, merging is not profitable whenever search costs and the number of competitors is sufficiently high.

(c) To prove this, we show that in the limit when $\bar{x} \rightarrow 1$ (or search costs go to zero), $\hat{\pi}^{*} / k-\pi^{*}>0$. Notice that for this it suffices to show that

$$
\lim _{\bar{x} \rightarrow 1}\left[\hat{\pi}^{*} / k-\frac{\tilde{p}^{*}}{n}\left(1-\tilde{p}^{* n}\right)\right]>0,
$$

because in the limit when $\bar{x} \rightarrow 1, \frac{\tilde{p}^{*}}{n}\left(1-\tilde{p}^{* n}\right)>\frac{p^{*}}{n}\left(1-p^{* n}\right)$.

Observe that equilibrium profits of the merging firms are

$$
\hat{\pi}^{*}=\hat{p}^{*}\left[\left(\bar{x}-\hat{p}^{*}+\tilde{p}^{*}\right)^{n-k}\left(1-\bar{x}^{k}\right)+k \int_{0}^{\bar{x}-\hat{p}^{*}}\left(\varepsilon+\tilde{p}^{*}\right)^{n-k}\left(\varepsilon+\hat{p}^{*}\right)^{k-1} d \varepsilon\right]
$$

Taking the derivative of $\hat{\pi}^{*}$ with respect to $\hat{p}^{*}$ and taking the limit when $\bar{x} \rightarrow 1$ gives

$$
-\left(1-\hat{p}^{*}+\tilde{p}^{*}\right)^{n-k} k \hat{p}^{*}+k \int_{0}^{1-\hat{p}^{*}}\left(\varepsilon+\tilde{p}^{*}\right)^{n-k}\left(\varepsilon+\hat{p}^{*}\right)^{k-2}\left(\varepsilon+k \hat{p}^{*}\right) d \varepsilon .
$$

This expression is identical to the FOC (12) when $\bar{x} \rightarrow 1$. As a result, for any value of $\hat{p}<\hat{p}^{*}$ (where $\hat{p}^{*}$ is the equilibrium price of a merging firm when $\bar{x} \rightarrow 1$ ), this derivative is negative. Since the equilibrium price of a non-merging firm is lower than the price of a merging one, we have

$$
\begin{aligned}
\lim _{\bar{x} \rightarrow 1} \hat{\pi}^{*} & =\lim _{\bar{x} \rightarrow 1}\left[\hat{p}^{*} k \int_{0}^{1-\hat{p}^{*}}\left(\varepsilon+\tilde{p}^{*}\right)^{n-k}\left(\varepsilon+\hat{p}^{*}\right)^{k-1} d \varepsilon\right]>\lim _{\bar{x} \rightarrow 1}\left[\tilde{p}^{*} k \int_{0}^{1-\tilde{p}^{*}}\left(\varepsilon+\tilde{p}^{*}\right)^{n-k}\left(\varepsilon+\tilde{p}^{*}\right)^{k-1} d \varepsilon\right] \\
& =\lim _{\bar{x} \rightarrow 1} \frac{k \tilde{p}^{*}}{n}\left(1-\tilde{p}^{* n}\right)>\lim _{\bar{x} \rightarrow 1} k \pi^{*} .
\end{aligned}
$$

where the last inequality follows from the observations that $\tilde{p}^{*}\left(1-\tilde{p}^{* n}\right)$ increases in $\tilde{p}^{*}$ and $\tilde{p}^{*}>p^{*}$. The result then follows.

Proof of Proposition 5. We start by deriving the payoffs of the firms in the situation where consumers start searching at the merging stores. As above, let $\hat{p}^{*}$ and $\tilde{p}^{*}$ denote the equilibrium prices of merging and non-merging stores, respectively. Consider first the payoff of the merging stores when they deviate by charging a price $\hat{p} \neq \hat{p}^{*}$. W.l.o.g. assume the merging stores are visited in a particular order, say, first the merging store 1 , then the merging store 2 etc. all the way till the merging store $k$. Consider a consumer who starts searching and visits the first merging store. If the match value there is less than $\bar{x}-\hat{p}^{*}+\hat{p}$, the consumer will continue searching and visit a second merging firm. Otherwise, the buyer will acquire right away the product sold there. In the second shop, and all the way till the $(k-1)^{t h}$ store the tradeoff faced by the consumer is exactly the same. Therefore, the direct demand obtained by the first $k-1$ merged stores is

$$
\sum_{i=1}^{k-1}\left(\bar{x}-\hat{p}^{*}+\hat{p}\right)^{i-1}\left(1-\bar{x}+\hat{p}^{*}-\hat{p}\right)=1-\left(\bar{x}-\hat{p}^{*}+\hat{p}\right)^{k-1}
$$

When a consumer arrives at the $k^{\text {th }}$ merging firm, the tradeoff a consumer faces is different because the firm to be visited next is a non-merging firm and this type of firm charges a different price, namely 
$\tilde{p}^{*}$. As a result, the consumer will search beyond the last merging firm if the highest observed utility is less than $\bar{x}-\tilde{p}^{*}$. In addition, we note that the consumer left the other $k-1$ shops of the merged entity because the utility levels there were less than $\bar{x}-\hat{p}^{*}$. Since $\hat{p}^{*}<\tilde{p}^{*}$ by assumption, this implies that some consumers may decide to return from the $k^{\text {th }}$ merging firm to one of the other merging firms. Denoting the fraction of consumers who return to a previously visited merging store without visiting all shops in the market by $\hat{r}_{m}$, we have:

$$
\begin{aligned}
\hat{r}_{m} & \equiv(k-1) \operatorname{Pr}\left[\bar{x}-\hat{p}^{*}>z_{k-1}-\hat{p}>\max \left\{\bar{x}-\tilde{p}^{*} ; \varepsilon_{k}-\hat{p}\right\}\right] \\
& =\frac{k-1}{k}\left[\left(\bar{x}-\hat{p}^{*}+\hat{p}\right)^{k}-\left(\bar{x}-\tilde{p}^{*}+\hat{p}\right)^{k}\right]
\end{aligned}
$$

The sub-index $m$ refers to the fact that these consumers return to a merging store after visiting only the merging stores.

A consumer terminates her search at the last merging store if the match value there is higher than at the other merging stores and it is not worth to continue searching further at the non-merging stores. This happens with probability

$$
\begin{aligned}
& \operatorname{Pr}\left[\varepsilon_{k}-\hat{p}>\max \left\{\bar{x}-\tilde{p}^{*} ; z_{k-1}-\hat{p}\right\}\right] \\
& =\left(\bar{x}-\hat{p}^{*}+\hat{p}\right)^{k-1}\left(1-\left(\bar{x}-\hat{p}^{*}+\hat{p}\right)\right)+\frac{1}{k}\left[\left(\bar{x}-\hat{p}^{*}+\hat{p}\right)^{k}-\left(\bar{x}-\tilde{p}^{*}+\hat{p}\right)^{k}\right]
\end{aligned}
$$

Finally, some consumers visit all shops in the market and return to one of the merging stores to conduct a purchase. This fraction of consumers, which we denote $\hat{r}_{a}$ to refer to the situation that consumers come back to one of the merging stores after visiting all firms in the market, is given by:

$$
\begin{aligned}
\hat{r}_{a} & \equiv \operatorname{Pr}\left[\bar{x}-\tilde{p}^{*}>z_{k}-\hat{p}>\max \left\{0 ; z_{n-k}-\tilde{p}^{*}\right\}\right] \\
& =k \int_{0}^{\bar{x}-\tilde{p}^{*}}(\varepsilon+\hat{p})^{k-1}\left(\varepsilon+\tilde{p}^{*}\right)^{n-k} d \varepsilon
\end{aligned}
$$

Putting the different demand terms together, the joint payoff function of the merging stores equals

$$
\hat{\pi}(\hat{p})=\hat{p}\left[1-\left(\bar{x}-\tilde{p}^{*}+\hat{p}\right)^{k}+k \int_{0}^{\bar{x}-\tilde{p}^{*}}(\varepsilon+\hat{p})^{k-1}\left(\varepsilon+\tilde{p}^{*}\right)^{n-k} d \varepsilon\right]
$$

Taking the FOC and imposing the equilibrium requirement that consumer expectations are correct we obtain the following condition:

$$
1-\left(\bar{x}-\tilde{p}^{*}+\hat{p}^{*}\right)^{k}-k\left(\bar{x}-\tilde{p}^{*}+\hat{p}^{*}\right)^{k-1} \hat{p}^{*}+k \int_{0}^{\bar{x}-\tilde{p}^{*}}\left(\varepsilon+\hat{p}^{*}\right)^{k-2}\left(\varepsilon+\tilde{p}^{*}\right)^{n-k}\left(\varepsilon+k \hat{p}^{*}\right) d \varepsilon=0
$$

Consider now the payoff function of a non-merging firm that deviates by charging a price $\tilde{p} \neq \tilde{p}^{*}$. Since consumers expect that all non-merging firms charge the same price $\tilde{p}^{*}$, we assume that they sample the non-merging firms in a random way. Thus, the probability that a typical non-merging firm is visited first, second and so on till the position $n-k$ equals $1 /(n-k)$. Note that conditional on arriving at a non-merging store, the probability that the buyer terminates her search there is $1-\bar{x}+\tilde{p}^{*}-\tilde{p}$. Consider a consumer who has visited all the merging stores and $h-1$ non-merging ones. Note that the consumer 
walked away from the merging stores because the highest match value there was lower than $\bar{x}-\tilde{p}^{*}+\hat{p}^{*}$; likewise, the consumer left the other non-merging stores because the match values there were lower than $\bar{x}$. Then the direct demand of a non-merging store when it is visited in $h^{\text {th }}$ place equals:

$$
\frac{1}{n-k} \bar{x}^{h-1}\left(\bar{x}-\tilde{p}^{*}+\hat{p}^{*}\right)^{k}\left(1-\bar{x}+\tilde{p}^{*}-\tilde{p}\right)
$$

Since the non-merging firm may be sampled in any position between 1 and $n-k$, its direct demand equals

$$
\sum_{h=1}^{n-k} \frac{1}{n-k} \bar{x}^{h-1}\left(\bar{x}-\tilde{p}^{*}+\hat{p}^{*}\right)^{k}\left(1-\bar{x}+\tilde{p}^{*}-\tilde{p}\right)=\frac{\left(\bar{x}-\tilde{p}^{*}+\hat{p}^{*}\right)^{k}}{n-k} \frac{1-\bar{x}^{n-k}}{1-\bar{x}}\left(1-\bar{x}+\tilde{p}^{*}-\tilde{p}\right)
$$

A non-merging store also obtains demand from consumers who visit all sellers in the market and return to it to conduct a purchase. Denoting this demand as $\tilde{r}_{a}$ to refer to the fact that these consumers return to a non-merging firm after visiting all other firms in the market we have:

$$
\begin{aligned}
\tilde{r}_{a} & \equiv \operatorname{Pr}\left[\max \left\{0 ; z_{k}-\hat{p}^{*} ; z_{n-k-1}-\tilde{p}^{*}\right\}<\varepsilon_{i}-\tilde{p}<\bar{x}-\tilde{p}^{*}\right] \\
& =\int_{0}^{\bar{x}-\tilde{p}^{*}}\left(\varepsilon+\hat{p}^{*}\right)^{k}\left(\varepsilon+\tilde{p}^{*}\right)^{n-k-1} d \varepsilon
\end{aligned}
$$

Putting together the various sources of demand of a non-merging firm, we obtain its payoff function:

$$
\tilde{\pi}=\tilde{p}\left[\frac{\left(\bar{x}-\tilde{p}^{*}+\hat{p}^{*}\right)^{k}}{n-k} \frac{1-\bar{x}^{n-k}}{1-\bar{x}}\left(1-\bar{x}+\tilde{p}^{*}-\tilde{p}\right)+\tilde{r}_{a}\right]
$$

The corresponding FOC is:

$$
\frac{\left(\bar{x}-\tilde{p}^{*}+\hat{p}^{*}\right)^{k}}{n-k} \frac{1-\bar{x}^{n-k}}{1-\bar{x}}\left(1-\bar{x}-\tilde{p}^{*}\right)+\int_{0}^{\bar{x}-\tilde{p}^{*}}\left(\varepsilon+\hat{p}^{*}\right)^{k}\left(\varepsilon+\tilde{p}^{*}\right)^{n-k-1} d \varepsilon=0
$$

If an equilibrium exists, the equilibrium prices $\hat{p}^{*}$ and $\tilde{p}^{*}$ must be given by the system of equations (38) and (39).

(a) We now prove that when search cost is sufficiently high then $\hat{p}^{*}>\tilde{p}^{*}$ and therefore consumer expectations are violated. We start by noting that, because the price of the non-merging firms is less than or equal to $1 / 2<p_{k}^{m}$ for all $\bar{x} \in\left[p_{k}^{m} ; 1\right]$, the integral in $(38)$ is positive. As a result, for an equilibrium to exist, the rest of the LHS of $(38), 1-\left(\bar{x}-\tilde{p}^{*}+\hat{p}^{*}\right)^{k}-k\left(\bar{x}-\tilde{p}^{*}+\hat{p}^{*}\right)^{k} \hat{p}^{*}$, must be negative. Note that this expression decreases in $\hat{p}^{*}$. Then, it must be higher than when we set $\hat{p}^{*}=\tilde{p}^{*}$ because $\hat{p}^{*}<\tilde{p}^{*}$ by assumption. That is, it must be the case that

$$
1-\left(\bar{x}-\tilde{p}^{*}+\hat{p}^{*}\right)^{k}-k\left(\bar{x}-\tilde{p}^{*}+\hat{p}^{*}\right)^{k} \hat{p}^{*}>1-\bar{x}^{k}-k \bar{x}^{k-1} \tilde{p}^{*}
$$

We now note that when $\bar{x} \rightarrow p_{k}^{m}$ the expression $1-\bar{x}^{k}-k \bar{x}^{k-1} p_{k}^{m}$ is equal to zero. Since $\tilde{p}^{*} \leq 1 / 2<p_{k}^{m}$, it is clear that $1-\bar{x}^{k}-k \bar{x}^{k-1} p_{k}^{m}>0$ when $\bar{x} \rightarrow p_{k}^{m}$. But this constitutes a contradiction because then the LHS of (38) cannot be negative. As a result, there is no such pair of prices $\hat{p}^{*}$ and $\tilde{p}^{*}$ that satisfy (38) and (39) when $\bar{x} \rightarrow p_{k}^{m}$ and $\hat{p}^{*}<\tilde{p}^{*}$.

(b) We prove now that when search cost goes to zero again we obtain $\hat{p}^{*}>\tilde{p}^{*}$, which violates consumer expectations. To show this we use the following equality

$$
Z \equiv Q-\hat{q}^{*}-(n-k) \tilde{q}^{*}=0
$$


where $Q=1-\hat{p}^{* k} \tilde{p}^{* n-k}$ denotes the aggregate quantity sold in the market and $\hat{q}^{*}$ and $\tilde{q}^{*}$ denote the equilibrium quantities of the merged entity and the non-merging firms. From the FOCs, these quantities are given by

$$
\begin{aligned}
& \hat{q}^{*}=k\left(\bar{x}-\tilde{p}^{*}+\hat{p}^{*}\right)^{k-1} \hat{p}^{*}-k(k-1) \hat{p}^{*} \int_{0}^{\bar{x}-\tilde{p}^{*}}\left(\varepsilon+\hat{p}^{*}\right)^{k-2}\left(\varepsilon+\tilde{p}^{*}\right)^{n-k} d \varepsilon \\
& \tilde{q}^{*}=\frac{\left(\bar{x}-\tilde{p}^{*}+\hat{p}^{*}\right)^{k-1}}{n-k} \frac{1-\bar{x}^{n-k}}{1-\bar{x}} \tilde{p}^{*}
\end{aligned}
$$

The partial derivative of $Z$ with respect to $\hat{p}^{*}$ is negative because $\tilde{q}^{*}$ increases with $\hat{p}^{*}$ and the derivative of $\hat{q}^{*}$ with respect to $\hat{p}^{*}$ is positive:

$$
\begin{aligned}
\frac{\partial \hat{q}^{*}}{\partial \hat{p}^{*}} & =k\left(\bar{x}-\tilde{p}^{*}+\hat{p}^{*}\right)^{k-1}+k(k-1)\left(\bar{x}-\tilde{p}^{*}+\hat{p}^{*}\right)^{k-2} \hat{p}^{*}-k(k-1) \int_{0}^{\bar{x}-\tilde{p}^{*}}\left(\varepsilon+\hat{p}^{*}\right)^{k-2}\left(\varepsilon+\tilde{p}^{*}\right)^{n-k} d \varepsilon \\
& -k(k-1)(k-2) \hat{p}^{*} \int_{0}^{\bar{x}-\tilde{p}^{*}}\left(\varepsilon+\hat{p}^{*}\right)^{k-3}\left(\varepsilon+\tilde{p}^{*}\right)^{n-k} d \varepsilon \\
& >k\left(\bar{x}-\tilde{p}^{*}+\hat{p}^{*}\right)^{k-1}+k(k-1)\left(\bar{x}-\tilde{p}^{*}+\hat{p}^{*}\right)^{k-2} \hat{p}^{*}-k(k-1) \bar{x}^{n-k} \int_{0}^{\bar{x}-\tilde{p}^{*}}\left(\varepsilon+\hat{p}^{*}\right)^{k-2} d \varepsilon \\
& -k(k-1)(k-2) \hat{p}^{*} \bar{x}^{n-k} \int_{0}^{\bar{x}-\tilde{p}^{*}}\left(\varepsilon+\hat{p}^{*}\right)^{k-3} d \varepsilon \\
& =k\left(\bar{x}-\tilde{p}^{*}+\hat{p}^{*}\right)^{k-2}\left(\bar{x}-\tilde{p}^{*}+k \hat{p}^{*}\right)\left(1-\bar{x}^{n-k}\right)>0 .
\end{aligned}
$$

Therefore, given that $\hat{p}^{*}<\tilde{p}^{*}$, if we set $\hat{p}^{*}=\tilde{p}^{*}$ then $Z$ must be negative when $\bar{x} \rightarrow 1$. That is, it must be the case that

$$
\begin{aligned}
\left.\lim _{\bar{x} \rightarrow 1} Z\right|_{\hat{p}^{*}=\tilde{p}^{*}} & =\lim _{\bar{x} \rightarrow 1}\left[1-\tilde{p}^{* n}-k \tilde{p}^{*}+k(k-1) \tilde{p}^{*} \int_{0}^{1-\tilde{p}^{*}}\left(\varepsilon+\tilde{p}^{*}\right)^{n-2} d \varepsilon-(n-k) \tilde{p}^{*}\right] \\
& =\lim _{\bar{x} \rightarrow 1}\left[1-\tilde{p}^{* n}-n \tilde{p}^{*}+\frac{k(k-1) \tilde{p}^{*}}{n-1}\left(1-\tilde{p}^{* n-1}\right)\right]<0 .
\end{aligned}
$$

The FOC (39) may be rearranged as

$$
1-\bar{x}-\tilde{p}^{*}+\frac{(n-k)(1-\bar{x})}{1-\bar{x}^{n-k}} \frac{1}{\left(\bar{x}-\tilde{p}^{*}+\hat{p}^{*}\right)^{k}} \int_{0}^{\bar{x}-\tilde{p}^{*}}\left(\varepsilon+\tilde{p}^{*}\right)^{n-k-1}\left(\varepsilon+\hat{p}^{*}\right)^{k} d \varepsilon=0
$$

The LHS of (42) increases in $\hat{p}^{*}$ because

$$
\begin{aligned}
& \frac{k\left(\bar{x}-\tilde{p}^{*}+\hat{p}^{*}\right)^{k} \int_{0}^{\bar{x}-\tilde{p}^{*}}\left(\varepsilon+\tilde{p}^{*}\right)^{n-k-1}\left(\varepsilon+\hat{p}^{*}\right)^{k-1} d \varepsilon-k\left(\bar{x}-\tilde{p}^{*}+\hat{p}^{*}\right)^{k-1} \int_{0}^{\bar{x}-\tilde{p}^{*}}\left(\varepsilon+\tilde{p}^{*}\right)^{n-k-1}\left(\varepsilon+\hat{p}^{*}\right)^{k} d \varepsilon}{\left(\bar{x}-\tilde{p}^{*}+\hat{p}^{*}\right)^{2 k}} \\
& =\frac{k\left(\bar{x}-\tilde{p}^{*}+\hat{p}^{*}\right) \int_{0}^{\bar{x}-\tilde{p}^{*}}\left(\varepsilon+\tilde{p}^{*}\right)^{n-k-1}\left(\varepsilon+\hat{p}^{*}\right)^{k-1} d \varepsilon-k \int_{0}^{\bar{x}-\tilde{p}^{*}}\left(\varepsilon+\tilde{p}^{*}\right)^{n-k-1}\left(\varepsilon+\hat{p}^{*}\right)^{k} d \varepsilon}{\left(\bar{x}-\tilde{p}^{*}+\hat{p}^{*}\right)^{k+1}} \\
& =\frac{k \int_{0}^{\bar{x}-\tilde{p}^{*}}\left(\varepsilon+\tilde{p}^{*}\right)^{n-k-1}\left(\varepsilon+\hat{p}^{*}\right)^{k-1}\left(\bar{x}-\tilde{p}^{*}+\hat{p}^{*}-\varepsilon-\hat{p}^{*}\right) d \varepsilon}{\left(\bar{x}-\tilde{p}^{*}+\hat{p}^{*}\right)^{k+1}} \\
& =\frac{k \int_{0}^{\bar{x}-\tilde{p}^{*}}\left(\varepsilon+\tilde{p}^{*}\right)^{n-k-1}\left(\varepsilon+\hat{p}^{*}\right)^{k-1}\left(\bar{x}-\tilde{p}^{*}-\varepsilon\right) d \varepsilon}{\left(\bar{x}-\tilde{p}^{*}+\hat{p}^{*}\right)^{k+1}}>0
\end{aligned}
$$


Therefore, given that $\hat{p}^{*}<\tilde{p}^{*}$, if we set $\hat{p}^{*}=\tilde{p}^{*}$ then the LHS of (42) must be positive, that is,

$$
1-\bar{x}-\tilde{p}^{*}+\frac{(n-k)(1-\bar{x})}{1-\bar{x}^{n-k}} \frac{1}{\bar{x}^{k}} \frac{1}{n}\left(\bar{x}^{n}-\tilde{p}^{* n}\right)>0
$$

If we take the limit of the LHS of (43) when $\bar{x} \rightarrow 1$, then we get the following inequality

$$
\lim _{\bar{x} \rightarrow 1}\left[-\tilde{p}^{*}+\frac{1}{n}\left(1-\tilde{p}^{* n}\right)\right]>0
$$

This inequality implies that $1-\tilde{p}^{* n}-n \tilde{p}^{*}>0$ in the limit when $\bar{x} \rightarrow 1$. This implies that (41) is positive. But this constitutes a contradiction and therefore it cannot be the case that $\hat{p}^{*}>\tilde{p}^{*}$ when $\bar{x} \rightarrow 1$.

(c) Now we prove that $\hat{p}^{*}>\tilde{p}^{*}$ if $n=3$. We will use the results from the proof of part (b) of this proposition. If $n=3$ then $Z\left(\tilde{p}^{*}\right)$ simplifies to

$$
Z_{\hat{p}^{*}=\tilde{p}^{*}}=1-\tilde{p}^{* 3}-2 \bar{x} \tilde{p}^{*}+\tilde{p}^{*}\left(\bar{x}^{2}-\tilde{p}^{* 2}\right)-\bar{x} \tilde{p}^{*}=1-2 \tilde{p}^{* 3}+\left(\bar{x}^{2}-3 \bar{x}\right) \tilde{p}^{*}
$$

while condition (43) reduces to

$$
1-\bar{x}-\tilde{p}^{*}+\frac{1}{3 \bar{x}^{2}}\left(\bar{x}^{3}-\tilde{p}^{* 3}\right)>0
$$

or

$$
\tilde{p}^{*}<1-\bar{x}+\frac{1}{3 \bar{x}^{2}}\left(\bar{x}^{3}-\tilde{p}^{* 3}\right)
$$

Then,

$$
\begin{aligned}
Z_{\hat{p}^{*}=\tilde{p}^{*}} & >1-2 \tilde{p}^{* 3}+\left(\bar{x}^{2}-3 \bar{x}\right)\left(1-\bar{x}+\frac{\bar{x}^{3}-\tilde{p}^{* 3}}{3 \bar{x}^{2}}\right)=1+\tilde{p}^{* 3} \frac{3-7 \bar{x}}{3 \bar{x}}-\frac{\bar{x}}{3}(3-\bar{x})(3-2 \bar{x}) \\
& >1+\left(\frac{1}{2}\right)^{3} \frac{3-7 \bar{x}}{3 \bar{x}}-\frac{\bar{x}}{3}(3-\bar{x})(3-2 \bar{x})=\frac{1}{24 \bar{x}}\left(3+17 \bar{x}-72 \bar{x}^{2}+72 \bar{x}^{3}-16 \bar{x}^{4}\right)
\end{aligned}
$$

which is always positive as shown in Figure 8.

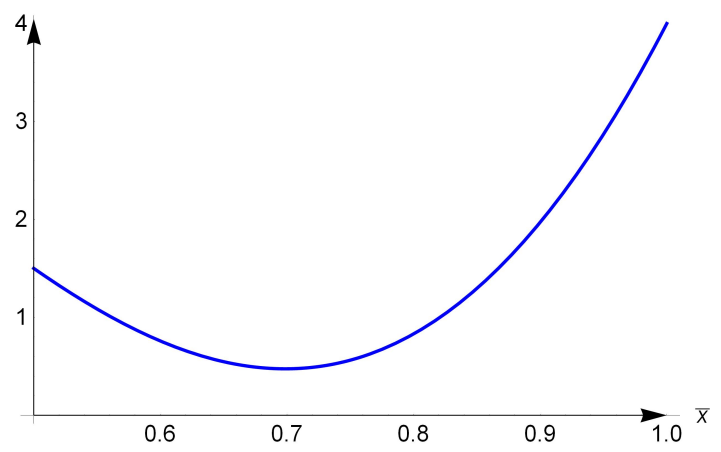

Figure 8: Plot of expression 44

Therefore, $Z_{\hat{p}^{*}=\tilde{p}^{*}}>0$ and $\hat{p}^{*}>\tilde{p}^{*}$ if $n=3$.

(d) Finally, in the limit when $n \rightarrow \infty$ the FOC of the merged entity becomes

$$
1-\left(\bar{x}-\tilde{p}^{*}+\hat{p}^{*}\right)^{k}-k\left(\bar{x}-\tilde{p}^{*}+\hat{p}^{*}\right)^{k-1} \hat{p}^{*}=0
$$


while that of a non-merging firm becomes

$$
\frac{1}{1-\bar{x}}\left(\bar{x}-\hat{p}^{*}+\tilde{p}^{*}\right)^{k}\left(1-\bar{x}-\tilde{p}^{*}\right)=0 .
$$

This implies that $\lim _{n \rightarrow \infty} \tilde{p}^{*}=1-\bar{x}$.

The LHS of (45) decreases in $\hat{p}^{*}$. Then, if $\hat{p}^{*}<\tilde{p}^{*}$, the LHS of (45) must be negative if we replace $\hat{p}^{*}$ by $\tilde{p}^{*}=1-\bar{x}$. However,

$$
1-\bar{x}^{k}-k \bar{x}^{k-1}(1-\bar{x})=1+(k-1) \bar{x}^{k}-k \bar{x}^{k-1} \geq 0
$$

where the inequality follows from setting $\bar{x}=1$. This establishes a contradiction so $\hat{p}^{*}<\tilde{p}^{*}$ cannot hold in the limit when $n \rightarrow \infty$. 


\section{References}

[1] Anderson, S. P. and R. Renault: "Pricing, Product Diversity, and Search Costs: a BertrandChamberlin-Diamond Model", RAND Journal of Economics 30, 719-35, 1999.

[2] Arbatskaya, M.: "Ordered Search“,RAND Journal of Economics 38, 119-127, 2007.

[3] Armstrong, M., J. Vickers, and J. Zhou: "Prominence and Consumer Search",RAND Journal of Economics, 40-2, 209-33, 2009.

[4] Deneckere, R. and C. Davidson: "Incentives to Form Coalitions with Bertrand Competition," RAND Journal of Economics 16, 473-86, 1985.

[5] Haan, M. A. and J. L. Moraga-González: "Advertising for Attention in a Consumer Search Model", The Economic Journal, 121, 552-579.

[6] Hortaçsu, A. and C. Syverson: "Product Differentiation, "Search Costs, and Competition in the Mutual Fund Industry: a Case Study of S\&P 500 Index Funds," The Quarterly Journal of Economics 119, 403-56, 2004.

[7] Janssen, M. C. W. and J. L. Moraga-González: “ On Mergers in Consumer Search Markets,” Tinbergen Institute Discussion Paper \# 2007-054/1, The Netherlands, 2007.

[8] Kohn, M.G. and S. Shavell: "The Theory of Search," Journal of Economic Theory 9, 93-123, 1974.

[9] Moraga-González, J. L. and V. Petrikaitè, "Economies of Search," unpublished manuscript, 2011.

[10] Salant, S. W., S. Switzer and R. J. Reynolds: "Losses from Horizontal Mergers: The Effects of Exogenous Change in Industry Structure on Cournot-Nash Equililibrium." Quarterly Journal of Economics 98: 185-99, 1983

[11] Weitzman, M. L.: "Optimal Search for the Best Alternative," Econometrica 47-3, 641-654, 1979.

[12] Wolinsky, A.: "True Monopolistic Competition as a Result of Imperfect Information," Quarterly Journal of Economics 101-3, 493-511, 1986.

[13] Zhou, J.: "Ordered Search in Differentiated Markets," International Journal of Industrial Organization 29-2, 253-262, 2011.

[14] Zhou, J.: "Prominence and Consumer Search: The Case of Multiple Prominent Firms," MPRA paper No. 12.554, 2009. 